\title{
Cyclic (Alkyl)(amino)carbene Lanthanide Amides: Synthesis, Structure and Catalytic Selective Hydrosilylation of Alkenes
}

Zexiong Pan, Jianying Zhang, Lulu Guo, Hao Yang, Jianfeng Li*, Chunming Cui*

State Key Laboratory of Elemento-Organic Chemistry and College of Chemistry, Nankai University, Tianjin 300071, China

E-mail: 1ijf@nankai.edu.cn, cmcui@nankai.edu.cn

Homepage: http://cui.nankai.edu.cn 


\section{Table of Contents}

$\begin{array}{ll}\text { General Experimental } & \text { S3 }\end{array}$

$\begin{array}{ll}\text { X-ray Diffraction Parameters and Data } & \text { S8 }\end{array}$

$\begin{array}{lr}\text { DFT Calculations } & \mathbf{S 1 0}\end{array}$

$\begin{array}{lr}\text { References } & \text { S15 }\end{array}$

$\begin{array}{lr}\text { GC-MS Spectra of Crude Reaction Mixtures } & \text { S18 }\end{array}$

$\begin{array}{lr}\text { NMR Spectra } & \text { S24 }\end{array}$ 


\section{General Experimental}

All manipulations involving air- and moisture-sensitive complexes were carried out under an atmosphere of dry argon by using modified Schlenk line and glovebox techniques. The melting point of the complexes were determined by Buchi M-560 melting point meter. Elemental analyses were carried out on an Elementar Vario EL analyzer. GC-MS data were obtained with a Thermo Scientific TRACE 1300 \& ISQ QD system, equipped with a TG-5MS column. The ${ }^{1} \mathrm{H},{ }^{13} \mathrm{C}$, and ${ }^{29} \mathrm{Si} \mathrm{NMR}$ spectroscopic data were recorded on a Bruker AV400 spectrometer. The ${ }^{13} \mathrm{C}$ NMR spectroscopic data of complex 2 was recorded on a Bruker AVANCE NEO $800 \mathrm{MHz}$ spectrometer. UV-visible absorption spectra were collected using a Shimadzu UV-2450 spectrophotometer.

Toluene and $n$-hexane were freshly distilled from sodium and degassed prior to use. $(\mathrm{THF})_{2} \mathrm{Yb}\left[\mathrm{N}\left(\mathrm{SiMe}_{3}\right)_{2}\right]_{2}{ }_{2} 1$ tetramethyl-2-pyrrolidinylidene $(\mathrm{CAAC}, \mathbf{1})^{\mathrm{S} 3}$ were synthesized according to the published procedures. Alkenes and silanes were vacuum-distilled over $\mathrm{CaH}_{2}$ and then were degassed prior to use. Other chemicals of analytical grade were used as received.

Synthesis of (CAAC)Yb[N(SiMe $\left.)_{2}\right]_{2}$ (2): After heating (THF) ${ }_{2} \mathrm{Yb}\left[\mathrm{N}\left(\mathrm{SiMe}_{3}\right)_{2}\right]_{2}(191 \mathrm{mg}, 0.3$ mmol, 1.0 equiv.) at $120^{\circ} \mathrm{C}$ in vacuum for $1 \mathrm{~h}$, the obtained $\mathrm{Yb}\left[\mathrm{N}\left(\mathrm{SiMe}_{3}\right)_{2}\right]_{2}$ was cooled down to room temperature, then adding $12 \mathrm{~mL}$ of $n$-hexane and cooling to $-78{ }^{\circ} \mathrm{C}$. A solution of CAAC $(85.6 \mathrm{mg}$, $0.3 \mathrm{mmol}, 1.0$ equiv.) in $8 \mathrm{~mL}$ of $n$-hexane was cooled to $-78^{\circ} \mathrm{C}$, then was added to the $\mathrm{Yb}\left[\mathrm{N}\left(\mathrm{SiMe}_{3}\right)_{2}\right]_{2}$ solution. The solution was allowed to warm to $0{ }^{\circ} \mathrm{C}$ and stirred for $18 \mathrm{~h}$. After filtration at $0{ }^{\circ} \mathrm{C}$, the solution was concentrated to $3 \mathrm{~mL}$. The dark green crystals of 2 (125 mg, 53\%) was afforded from $n$ hexane at $-40{ }^{\circ} \mathrm{C} . \mathbf{M p}: 110.9-112.8{ }^{\circ} \mathrm{C} .{ }^{1} \mathbf{H}$ NMR (400 MHz, C6 6 6, $\left.298 \mathrm{~K}\right) \delta 7.08$ (s, 1H, p-Ar- $H$ ), 6.96 (s, 2H, m-Ar- $H), 2.61$ (s, 2H, Ph- $\left.\mathrm{CH}\left(\mathrm{CH}_{3}\right)_{2}\right), 1.39$ (s, 6H, $\left.\mathrm{NC}\left(\mathrm{CH}_{3}\right)_{2}\right), 1.26$ (s, 6H, Ph-CH(CH3)2), 1.22 (s, 3H, $\left.\mathrm{Si}\left(\mathrm{CH}_{3}\right)_{3}\right), 1.04$ (s, 6H, Ph-CH(CH3)2), 0.88 (s, 2H, $\left.\mathrm{CH}_{2}\right), 0.81\left(\mathrm{~s}, 6 \mathrm{H}, \mathrm{NCC}\left(\mathrm{CH}_{3}\right)_{2}\right), 0.36$ (s, 30H, $\left.\mathrm{Si}\left(\mathrm{CH}_{3}\right)_{3}\right), 0.30$ (s, 3H, $\left.\mathrm{Si}\left(\mathrm{CH}_{3}\right)_{3}\right) .{ }^{13} \mathbf{C}\left\{{ }^{1} \mathbf{H}\right\}$ NMR $\left(202 \mathrm{MHz}, \mathrm{C}_{6} \mathrm{D}_{6}, 298 \mathrm{~K}\right) \delta 288.41$ (carbeneC), 144.95, 135.83, 129.47, 128.29, $125.21(\mathrm{Ar}-C), 83.36,56.77\left(C_{\mathrm{q}}\right), 50.43\left(\mathrm{CH}_{2}\right), 28.89\left(\mathrm{CH}\left(\mathrm{CH}_{3}\right)_{2}\right)$, 28.76, 28.24, 27.95, $23.71\left(\mathrm{CH}_{3}\right), 6.38\left(\mathrm{Si}\left(\mathrm{CH}_{3}\right)_{3}\right)$. Despite several attempts, no satisfying elemental analysis data of the low-coordinated complex 2 could be obtained due to its highly air- and moisturesensitive properties. 
Synthesis of (CAAC)Eu[N(SiMe $\left.)_{2}\right)_{2}$ (THF) (3): At $-78^{\circ} \mathrm{C}$, a solution of CAAC (143 $\mathrm{mg}, 0.5$ mmol, 1.0 equiv.) in $n$-hexane $(7 \mathrm{~mL})$ was added to a solution of $(\mathrm{THF})_{2} \mathrm{Eu}\left[\mathrm{N}\left(\mathrm{SiMe}_{3}\right)_{2}\right]_{2}(309 \mathrm{mg}, 0.5$ mmol, 1.0 equiv.) in $n$-hexane $(7 \mathrm{~mL})$. The solution was allowed to warm to room temperature and stirred for $12 \mathrm{~h}$. After filtration, the solution was concentrated to $3 \mathrm{~mL}$. The yellow crystals of $\mathbf{3}$ (205 mg, 49\%) was afforded from $n$-hexane at $-40{ }^{\circ} \mathrm{C}$. Mp: $90.5-92.9^{\circ} \mathrm{C}$. Anal. Calcd for $\mathrm{C}_{36} \mathrm{H}_{75} \mathrm{~N}_{3} \mathrm{OSSi}_{4} \mathrm{Eu}$ (830.32): C, 52.08; H, 9.10; N, 5.06. Found: C, 52.21; H, 8.99; N, 5.24.

Typical Procedure for Catalytic Hydrosilylation. In an Ar glove box, a Schlenk tube was charged with catalyst $(8.3 \mathrm{mg}, 0.010 \mathrm{mmol}, 1 \mathrm{~mol} \%)$ and $0.4 \mathrm{~mL}$ toluene. And then silane (1.2 mmol, 1.2 equiv.) and alkene (1.0 mmol, 1.0 equiv.) were added. The Schlenk tube was quickly removed from the glovebox. The reaction mixture was stirred at room temperature or $80^{\circ} \mathrm{C}$ using oil bath. Conversion and regioselectivity were analyzed by GC-MS of the crude reaction mixture after quenching and diluting the crude reaction mixture with $n$-hexane. After the reaction was complete, the product was purified by column chromatography on silica gel ( $n$-hexane for elution) to yield colorless oil. The spectra of catalytic hydrosilylation products $\mathbf{7} \mathbf{a}^{\mathrm{S} 4}, \mathbf{7} \mathbf{b}^{\mathrm{S} 5}, \mathbf{7} \mathbf{c}^{\mathrm{S} 5}, \mathbf{7} \mathbf{d}^{\mathrm{S} 6}, \mathbf{7} \mathbf{e}^{\mathrm{S} 7}, \mathbf{7} \mathbf{f}^{\mathrm{S} 4}, \mathbf{7} \mathbf{g}^{\mathrm{S} 5}, \mathbf{7} \mathbf{h}^{\mathrm{S} 4}, \mathbf{7} \mathbf{i}^{\mathrm{S} 7}, \mathbf{7} \mathbf{j}^{\mathrm{S} 4}, \mathbf{7} \mathbf{k}^{\mathrm{S} 4}$,

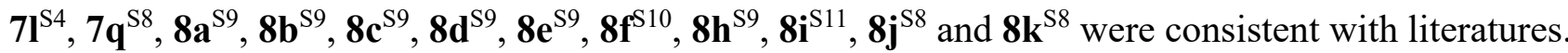

Table S1. Hydrosilylation reactions of styrene with various silanes. ${ }^{a}$

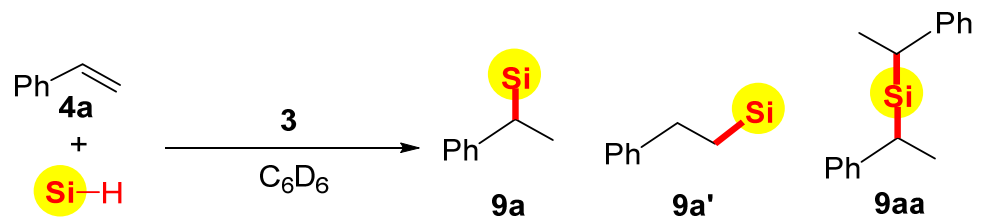

\begin{tabular}{ccccccc}
\hline entry & $\begin{array}{c}\text { loading } \\
\text { of } \mathbf{3}(\%)\end{array}$ & $\begin{array}{c}\mathrm{T} \\
\left({ }^{\circ} \mathrm{C}\right)\end{array}$ & $\begin{array}{c}\text { time } \\
(\mathrm{h})\end{array}$ & silane & $\begin{array}{c}\text { conv. } \\
(\%)^{b}\end{array}$ & $\mathbf{9 a} / \mathbf{9 a} \mathbf{a}^{\prime} / \mathbf{9 a a}$ \\
\hline 1 & 0.5 & 80 & 6 & $n-\mathrm{HexSiH}_{3}$ & 62 & $94 / 0 / 6$ \\
2 & 1 & 60 & 6 & $\mathrm{PhMeSiH}_{2}$ & 70 & $74 / 0 / 26$ \\
3 & 5 & 80 & 8 & $\mathrm{Et}_{2} \mathrm{SiH}_{2}$ & 0 & - \\
$\begin{array}{l}a \\
\text { conversions of styrene and selectivities were determined by GC-MS measurement of the crude products. }\end{array}$
\end{tabular}




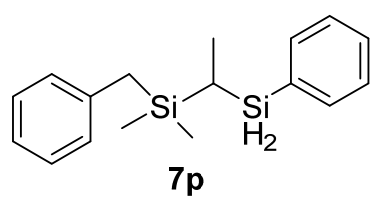

Following the general procedure, alkene (176 mg, $1.0 \mathrm{mmol}, 1.0$ equiv.) and $\mathrm{PhSiH}_{3}$ (130 mg, $1.2 \mathrm{mmol}, 1.2$ equiv.) was converted to the corresponding $\mathbf{7 p}$ (263 mg, yield $93 \%$, regioselectivity $>99 \%$ ) using $3(8.3 \mathrm{mg}, 0.010 \mathrm{mmol}, 1$ mol \%) at $80{ }^{\circ} \mathrm{C}$ for 6 h. ${ }^{1} \mathbf{H}$ NMR $\left(400 \mathrm{MHz}, \mathrm{CDCl}_{3}, 298 \mathrm{~K}\right) \delta 7.70-7.65$ (m, 2H, Ar- $\left.H\right), 7.51-7.42$ $(\mathrm{m}, 3 \mathrm{H}, \mathrm{Ar}-H), 7.31\left(\mathrm{t},{ }^{3} J_{\mathrm{HH}}=7.5 \mathrm{~Hz}, 2 \mathrm{H}, \mathrm{Ar}-H\right), 7.17\left(\mathrm{t},{ }^{3} J_{\mathrm{HH}}=7.4 \mathrm{~Hz}, 1 \mathrm{H}, \mathrm{Ar}-H\right), 7.10\left(\mathrm{~d},{ }^{3} J_{\mathrm{HH}}=7.2\right.$ $\mathrm{Hz}, 2 \mathrm{H}, \mathrm{Ar}-H), 4.54\left(\mathrm{~m}, 1 \mathrm{H}, \mathrm{Si} H_{2}\right), 4.37\left(\mathrm{~m}, 1 \mathrm{H}, \mathrm{Si} H_{2}\right), 2.25\left(\mathrm{~m}, 2 \mathrm{H}, \mathrm{PhCH}_{2} \mathrm{Si}\right), 1.24\left(\mathrm{~d},{ }^{3} \mathrm{~J}_{\mathrm{HH}}=7.5 \mathrm{~Hz}\right.$, $\left.3 \mathrm{H}, \mathrm{CHCH}_{3}\right), 0.43\left(\mathrm{~m}, 1 \mathrm{H}, \mathrm{CHCH}_{3}\right), 0.10\left(\mathrm{~s}, 3 \mathrm{H}, \mathrm{Si}\left(\mathrm{CH}_{3}\right)_{2}\right), 0.09\left(\mathrm{~s}, 3 \mathrm{H}, \mathrm{Si}\left(\mathrm{CH}_{3}\right)_{2}\right) .{ }^{13} \mathbf{C}\left\{{ }^{1} \mathbf{H}\right\} \mathbf{N M R}$ $\left(101 \mathrm{MHz}, \mathrm{CDCl}_{3}, 298 \mathrm{~K}\right) \delta 140.10,135.66,135.41,132.77,129.51,128.16,127.94,123.96(\mathrm{Ar}-C)$, $24.87\left(\mathrm{PhCH}_{2} \mathrm{Si}\right), 10.57\left(\mathrm{CHCH}_{3}\right), 0.59\left(\mathrm{CHCH}_{3}\right),-3.76\left(\mathrm{Si}\left(\mathrm{CH}_{3}\right)_{2}\right),-4.25\left(\mathrm{Si}\left(\mathrm{CH}_{3}\right)_{2}\right) .{ }^{29} \mathrm{Si} \mathrm{NMR}(79$ $\left.\mathrm{MHz}_{\mathrm{CDCl}}, 298 \mathrm{~K}\right) \delta 5.17\left(\mathrm{~s}, \mathrm{Si}\left(\mathrm{CH}_{3}\right)_{2}\right),-27.31\left(\mathrm{t},{ }^{1} J_{\mathrm{SiH}}=191 \mathrm{~Hz}, \mathrm{SiH}_{2}\right) . \mathbf{E I}-\mathbf{M S}$ for $\left\{[\mathrm{M}]^{+}\right.$, $\left.\left[\mathrm{C}_{17} \mathrm{H}_{24} \mathrm{Si}_{2}\right]^{+}\right\}: \mathrm{m} / \mathrm{z} 284.28$.

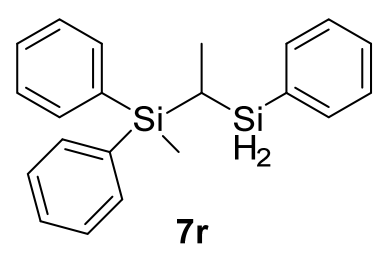

Following the general procedure, alkene ( $44.9 \mathrm{mg}, 0.2 \mathrm{mmol}, 1.0$ equiv.) and $\mathrm{PhSiH}_{3}$ (26.0 mg, $0.24 \mathrm{mmol}, 1.2$ equiv.) was converted to the corresponding 7r (59 mg, yield $89 \%$, regioselectivity $>99 \%$ ) using $3(8.3 \mathrm{mg}, 0.010 \mathrm{mmol}$, $5 \mathrm{~mol} \%)$ at $80{ }^{\circ} \mathrm{C}$ for 4 h. ${ }^{1} \mathbf{H}$ NMR $\left(400 \mathrm{MHz}, \mathrm{CDCl}_{3}, 298 \mathrm{~K}\right) \delta 7.68-7.58$ (m, 4H, Ar- $H$ ), $7.54\left(\mathrm{~d},{ }^{3} J_{\mathrm{HH}}=6.6 \mathrm{~Hz}, 2 \mathrm{H}, \mathrm{Ar}-H\right), 7.49-7.34(\mathrm{~m}, 9 \mathrm{H}, \mathrm{Ar}-H), 4.44\left(\mathrm{~m}, 1 \mathrm{H}, \mathrm{Si} H_{2}\right), 4.29$ $\left(\mathrm{m}, 1 \mathrm{H}, \mathrm{Si} H_{2}\right), 1.25\left(\mathrm{~d},{ }^{3} J_{\mathrm{HH}}=7.2 \mathrm{~Hz}, 3 \mathrm{H}, \mathrm{CHCH}_{3}\right), 1.01\left(\mathrm{~m}, 1 \mathrm{H}, \mathrm{CHCH}_{3}\right), 0.65\left(\mathrm{~s}, 3 \mathrm{H}, \mathrm{Si}\left(\mathrm{CH}_{3}\right)\right)$. ${ }^{13} \mathbf{C}\left\{{ }^{1} \mathbf{H}\right\}$ NMR $\left(101 \mathrm{MHz}, \mathrm{CDCl}_{3}, 298 \mathrm{~K}\right) \delta$ 136.59, 136.29, 135.37, 134.73, 132.64, 129.42, 129.16, 127.84, $127.75(\mathrm{Ar}-\mathrm{C}), 11.21\left(\mathrm{CHCH}_{3}\right), 0.14\left(\mathrm{CHCH}_{3}\right),-4.94\left(\mathrm{Si}\left(\mathrm{CH}_{3}\right)\right) .{ }^{29} \mathrm{Si} \mathbf{N M R}\left(79 \mathrm{MHz}, \mathrm{CDCl}_{3}\right.$, $298 \mathrm{~K}) \delta-4.75\left(\mathrm{~s}, \mathrm{Si}\left(\mathrm{CH}_{3}\right)\right),-26.64\left(\mathrm{t},{ }^{1} \mathrm{~J}_{\mathrm{SiH}}=196 \mathrm{~Hz}, \mathrm{SiH}_{2}\right)$. EI-MS for $\left\{[\mathrm{M}]^{+},\left[\mathrm{C}_{21} \mathrm{H}_{24} \mathrm{Si}_{2}\right]^{+}\right\}: \mathrm{m} / \mathrm{z}$ 332.24 .

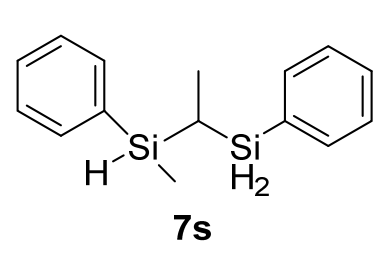

Following the general procedure, alkene (148 mg, $1.0 \mathrm{mmol}, 1.0$ equiv.) and $\mathrm{PhSiH}_{3}$ (130 mg, $1.2 \mathrm{mmol}, 1.2$ equiv.) was converted to the corresponding 7 s (230 mg, yield $90 \%$, regioselectivity $97 \%$ ) using 3 (8.3 mg, $0.010 \mathrm{mmol}$, $1 \mathrm{~mol} \%)$ at $80{ }^{\circ} \mathrm{C}$ for $4 \mathrm{~h} .{ }^{1} \mathbf{H}$ NMR $\left(400 \mathrm{MHz}, \mathrm{CDCl}_{3}, 298 \mathrm{~K}\right) \delta 7.66-7.58$ (m, 4H, Ar- $H$ ), 7.50-7.35 (m, 6H, Ar- $H$ ), 4.58-4.47 (m, 2H, Si $\left.H, \mathrm{Si} H_{2}\right), 4.37$ (m, 1H, Si $\left.H_{2}\right), 1.24$ (m, $\left.3 \mathrm{H}, \mathrm{CHCH}_{3}\right), 0.70\left(\mathrm{~m}, 1 \mathrm{H}, \mathrm{CHCH}_{3}\right), 0.45\left(\mathrm{~m}, 3 \mathrm{H}, \mathrm{Si}\left(\mathrm{CH}_{3}\right)\right) \cdot{ }^{13} \mathbf{C}\left\{{ }^{1} \mathbf{H}\right\} \mathbf{N M R}\left(101 \mathrm{MHz}, \mathrm{CDCl}_{3}, 298 \mathrm{~K}\right)$ $\delta 135.73,135.66,135.59,135.42,134.58,134.51 .132 .33,132.29,129.86,129.53,129.33,128.10$, 
127.90, $127.80(\mathrm{Ar}-\mathrm{C}), 11.36,10.70\left(\mathrm{CHCH}_{3}\right),-0.11,-0.45\left(\mathrm{CHCH}_{3}\right),-5.65,-6.88\left(\mathrm{Si}\left(\mathrm{CH}_{3}\right)\right) .{ }^{29} \mathrm{Si}$ NMR $\left(79 \mathrm{MHz}, \mathrm{CDCl}_{3}, 298 \mathrm{~K}\right) \delta-8.53\left(\mathrm{~d},{ }^{1} J_{\mathrm{SiH}}=185 \mathrm{~Hz}, \mathrm{SiH}\right),-10.54\left(\mathrm{~d},{ }^{1} J_{\mathrm{SiH}}=197 \mathrm{~Hz}, \mathrm{SiH}\right),-$ $26.32\left(\mathrm{t},{ }^{1} J_{\mathrm{SiH}}=201 \mathrm{~Hz}, \mathrm{SiH} 2\right)$. EI-MS for $\left\{[\mathrm{M}-\mathrm{H}]^{+},\left[\mathrm{C}_{15} \mathrm{H}_{19} \mathrm{Si}_{2}\right]^{+}\right\}: \mathrm{m} / \mathrm{z} 255.37$.

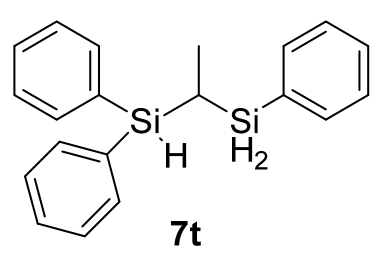

Following the general procedure, alkene $(210 \mathrm{mg}, 1.0 \mathrm{mmol}, 1.0$ equiv.) and $\mathrm{PhSiH}_{3}$ (130 mg, $1.2 \mathrm{mmol}, 1.2$ equiv.) was converted to the corresponding $7 \mathbf{t}$ (301 mg, yield $95 \%$, regioselectivity $96 \%$ ) using $3(8.3 \mathrm{mg}, 0.010 \mathrm{mmol}, 1$ mol \%) at $80{ }^{\circ} \mathrm{C}$ for 6 h. ${ }^{1} \mathbf{H}$ NMR $\left(400 \mathrm{MHz}, \mathrm{CDCl}_{3}, 298 \mathrm{~K}\right) \delta 7.66\left(\mathrm{~d},{ }^{3} \mathrm{~J}_{\mathrm{HH}}\right.$ $=6.5 \mathrm{~Hz}, 4 \mathrm{H}, \mathrm{Ar}-H), 7.57\left(\mathrm{~d},{ }^{3} J_{\mathrm{HH}}=6.6 \mathrm{~Hz}, 2 \mathrm{H}, \mathrm{Ar}-H\right), 7.50-7.35(\mathrm{~m}, 9 \mathrm{H}, \mathrm{Ar}-H), 4.99\left(\mathrm{~d},{ }^{3} J_{\mathrm{HH}}=3.7\right.$ $\mathrm{Hz}, 1 \mathrm{H}, \mathrm{Si} H), 4.49\left(\mathrm{~m}, 1 \mathrm{H}, \mathrm{Si} H_{2}\right), 4.36\left(\mathrm{~m}, 1 \mathrm{H}, \mathrm{Si} H_{2}\right), 1.30$ (d, $\left.{ }^{3} \mathrm{~J}_{\mathrm{HH}}=7.4 \mathrm{~Hz}, 3 \mathrm{H}, \mathrm{CHCH}_{3}\right), 1.05(\mathrm{~m}$, $\left.1 \mathrm{H}, \mathrm{CHCH}_{3}\right) .{ }^{13} \mathbf{C}\left\{{ }^{1} \mathbf{H}\right\}$ NMR $\left(101 \mathrm{MHz}, \mathrm{CDCl}_{3}, 298 \mathrm{~K}\right) \delta 135.44,135.32,133.86,133.53,132.12$, 129.61, 129.59, 129.52, $127.92,127.87(\mathrm{Ar}-\mathrm{C}), 11.39\left(\mathrm{CHCH}_{3}\right),-1.30\left(\mathrm{CHCH}_{3}\right) .{ }^{29} \mathrm{Si} \mathbf{N M R}(79 \mathrm{MHz}$, $\left.\mathrm{CDCl}_{3}, 298 \mathrm{~K}\right) \delta-10.14\left(\mathrm{~d},{ }^{1} J_{\mathrm{SiH}}=196 \mathrm{~Hz}, \mathrm{SiH}\right),-25.88\left(\mathrm{t},{ }^{1} J_{\mathrm{SiH}}=197 \mathrm{~Hz}, \mathrm{SiH}_{2}\right) . \mathbf{E I}-\mathbf{M S}$ for $\{[\mathrm{M}-$ $\left.\mathrm{H}]^{+},\left[\mathrm{C}_{20} \mathrm{H}_{21} \mathrm{Si}_{2}\right]^{+}\right\}: \mathrm{m} / \mathrm{z} 317.31$.

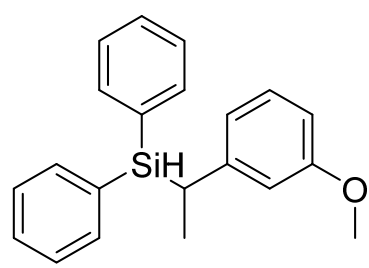

$8 \mathrm{~g}$

Following the general procedure, alkene (134 mg, $1.0 \mathrm{mmol}, 1.0$ equiv.) and $\mathrm{Ph}_{2} \mathrm{SiH}_{2}$ (221 mg, $1.2 \mathrm{mmol}, 1.2$ equiv.) was converted to the corresponding $\mathbf{8 g}$ (195 mg, yield $61 \%$, regioselectivity $>99 \%$ ) using 3 ( $8.3 \mathrm{mg}, 0.010 \mathrm{mmol}$, $1 \mathrm{~mol} \%$ ) at $80{ }^{\circ} \mathrm{C}$ for 12 h. ${ }^{1} \mathbf{H}$ NMR (400 MHz, $\left.\mathrm{CDCl}_{3}, 298 \mathrm{~K}\right) \delta 7.64(\mathrm{~d}$, $\left.{ }^{3} J_{\mathrm{HH}}=6.7 \mathrm{~Hz}, 2 \mathrm{H}, \mathrm{Ar}-H\right), 7.54-7.33(\mathrm{~m}, 8 \mathrm{H}, \mathrm{Ar}-H), 7.20\left(\mathrm{t},{ }^{3} J_{\mathrm{HH}}=7.8 \mathrm{~Hz}, 1 \mathrm{H}\right.$, $\operatorname{Ar}-H), 6.78-6.71(\mathrm{~m}, 2 \mathrm{H}, \mathrm{Ar}-H), 6.61(\mathrm{~s}, 1 \mathrm{H}, \mathrm{Ar}-H), 4.95\left(\mathrm{~d},{ }^{3} J_{\mathrm{HH}}=3.0 \mathrm{~Hz}, 1 \mathrm{H}, \mathrm{Si} H\right), 3.71(\mathrm{~s}, 3 \mathrm{H}$, $\left.\mathrm{OCH}_{3}\right), 2.92(\mathrm{~m}, 1 \mathrm{H}, \mathrm{CHCH} 3), 1.56\left(\mathrm{~d},{ }^{3} \mathrm{JHH}_{\mathrm{HH}}=7.5 \mathrm{~Hz}, 3 \mathrm{H}, \mathrm{CHCH}_{3}\right) .{ }^{13} \mathbf{C}\left\{{ }^{1} \mathbf{H}\right\} \mathbf{N M R}\left(101 \mathrm{MHz}, \mathrm{CDCl}_{3}\right.$, 298 K) $\delta 159.31,145.89,135.70,135.59,135.46,132.97,129.70,129.64,129.53,129.08,127.94$, 127.81, 127.62, 120.10, 113.05, 110.81 (Ar-C), $54.94\left(\mathrm{OCH}_{3}\right), 27.10\left(\mathrm{CHCH}_{3}\right), 16.35\left(\mathrm{CHCH}_{3}\right) .{ }^{29} \mathrm{Si}$ NMR $\left(79 \mathrm{MHz}, \mathrm{CDCl}_{3}, 298 \mathrm{~K}\right) \delta-8.33\left(\mathrm{~d},{ }^{1} \mathrm{~J}_{\mathrm{SiH}}=197 \mathrm{~Hz}, \mathrm{SiH}\right)$. EI-MS for $\left\{[\mathrm{M}]^{+},\left[\mathrm{C}_{21} \mathrm{H}_{22} \mathrm{OSi}\right]^{+}\right\}$: $\mathrm{m} / \mathrm{z} 318.36$. 


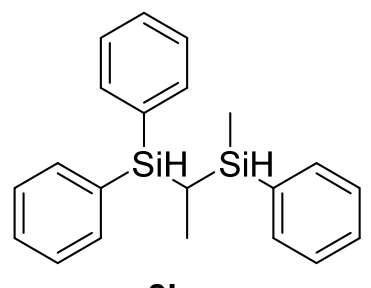

8I

Following the general procedure, alkene $(29.7 \mathrm{mg}, 0.20 \mathrm{mmol}, 1.0$ equiv.) and $\mathrm{Ph}_{2} \mathrm{SiH}_{2}$ (44.2 mg, $0.24 \mathrm{mmol}, 1.2$ equiv.) was converted to the corresponding 81 (50 mg, yield $75 \%$, regioselectivity $90 \%$ ) using 3 (8.3 mg, $0.010 \mathrm{mmol}, 5$ mol \%) at $80{ }^{\circ} \mathrm{C}$ for $24 \mathrm{~h} .{ }^{1} \mathbf{H}$ NMR $\left(400 \mathrm{MHz}, \mathrm{CDCl}_{3}, 298 \mathrm{~K}\right) \delta 7.67-7.61(\mathrm{~m}$, 4H, Ar-H), 7.57-7.51 (m, 2H, Ar-H), 7.46-7.35 (m, 9H, Ar- $H$ ), 5.00-4.94 (m, $1 \mathrm{H}, \mathrm{Ph}_{2} \mathrm{Si} H$ ), 4.54-4.42 (m, 1H, $\left.\mathrm{Si} H \mathrm{CH}_{3}\right), 1.22$ (m, 3H, $\left.\mathrm{CHCH}_{3}\right), 1.03-0.56\left(\mathrm{~m}, 1 \mathrm{H}, \mathrm{CHCH}_{3}\right), 0.30$ $\left(\mathrm{m}, 3 \mathrm{H}, \mathrm{SiHCH}_{3}\right) .{ }^{13} \mathbf{C}\left\{{ }^{1} \mathbf{H}\right\} \mathbf{N M R}\left(101 \mathrm{MHz}, \mathrm{CDCl}_{3}, 298 \mathrm{~K}\right) \delta 136.14,135.90,135.79,135.49,135.25$, $135.15,134.62,134.47,134.36,134.00,129.79,129.54,129.46,129.26,129.19,128.04,127.95$, $127.92,127.87,127.75,127.71,127.68(\mathrm{Ar}-\mathrm{C}), 10.82,9.96\left(\mathrm{CHCH}_{3}\right), 1.41,0.84\left(\mathrm{CHCH}_{3}\right),-5.10,-$ $7.06\left(\mathrm{SiHCH}_{3}\right) .{ }^{29} \mathrm{Si}$ NMR $\left(79 \mathrm{MHz}, \mathrm{CDCl}_{3}, 298 \mathrm{~K}\right) \delta-8.54\left(\mathrm{~d},{ }^{1} J_{\mathrm{SiH}}=199 \mathrm{~Hz}, \mathrm{Ph}_{2} \mathrm{SiH}\right),-11.32(\mathrm{~d}$, $\left.{ }^{1} J_{\mathrm{SiH}}=178 \mathrm{~Hz}, \mathrm{SiHCH}_{3}\right)$. EI-MS for $\left\{[\mathrm{M}]^{+},\left[\mathrm{C}_{21} \mathrm{H}_{24} \mathrm{Si}_{2}\right]^{+}\right\}: \mathrm{m} / \mathrm{z} 331.93$. 


\section{X-ray Diffraction Parameters and Data}

All intensity data were collected with a Bruker SMART CCD diffractometer, using graphitemonochromated Mo K $\alpha$ radiation $(\lambda=0.71073 \AA)$. The structures were resolved by direct methods and refined by full matrix least squares on $F^{2}{ }^{S 12}$ All the other hydrogen atoms were considered in calculated positions. All non-hydrogen atoms were refined anisotropically. The Ortep-3 program was utilized to draw the molecules. ${ }^{\mathrm{S} 13}$ Crystal data and data collection details are collected in Tables S2. CCDC 2064197 and 2068390 contain the supplementary crystallographic data for this paper. These data can be obtained free of charge from The Cambridge Crystallographic Data Centre via www.ccdc.cam.ac.uk/data_request/cif.

Table S2. Crystallographic detail for $\mathbf{2}$ and $\mathbf{3}$.

\begin{tabular}{ccc}
\hline & $\mathbf{2}$ & $\mathbf{3}$ \\
\hline formula & $\mathrm{C}_{32} \mathrm{H}_{67} \mathrm{~N}_{3} \mathrm{Si}_{4} \mathrm{Yb}$ & $\mathrm{C}_{36} \mathrm{H}_{75} \mathrm{EuN}_{3} \mathrm{OSi}_{4}$ \\
$\mathrm{FW}$ & 779.28 & 830.31 \\
$T(\mathrm{~K})$ & 113 & 113 \\
space group & $P b c a$ & $P 2 / n$ \\
$a(\AA)$ & $13.3527(3)$ & $12.517(3)$ \\
$b(\AA)$ & $21.5298(4)$ & $20.617(4)$ \\
$c(\AA)$ & $28.6350(6)$ & $17.818(4)$ \\
$\alpha\left({ }^{\circ}\right)$ & 90 & 90 \\
$\beta\left(^{\circ}\right)$ & 90 & $94.58(3)$ \\
$\gamma\left({ }^{\circ}\right)$ & 90 & 90 \\
$V\left(\AA^{3}\right)$ & $8232.0(3)$ & $4583.5(16)$ \\
$Z$ & 8 & 4 \\
$d_{\text {calcd }}\left(\mathrm{g} / \mathrm{cm}{ }^{3}\right)$ & 1.258 & 1.203 \\
$F(000)$ & 3248.0 & 1.080 \\
$\mathrm{GOF}$ & 1.039 & $0.0433,0.0926$ \\
$R_{1}, w R_{2}(I>2 \sigma(I))$ & $0.0265,0.0516$ & $0.0511,0.0968$ \\
$R_{1}, w R_{2}$ & $0.0347,0.0548$ & 2068390 \\
$($ all data $)$ & 2064197 & \\
$\mathrm{CCDC}$ & &
\end{tabular}


Table S3. Selected bond lengths $(\AA)$ and angles $\left(^{\circ}\right)$ in $\mathbf{2}$ and $\mathbf{3}$.

\begin{tabular}{cccc}
\hline & $\mathbf{2}$ & \multicolumn{2}{c}{$\mathbf{3}$} \\
\hline $\mathrm{Yb} 1-\mathrm{C} 1$ & $2.672(3)$ & $\mathrm{Eu} 1-\mathrm{C} 1$ & $2.945(3)$ \\
$\mathrm{Yb} 1-\mathrm{N} 2$ & $2.321(2)$ & $\mathrm{Eu} 1-\mathrm{N} 2$ & $2.460(3)$ \\
$\mathrm{Yb} 1-\mathrm{N} 3$ & $2.327(2)$ & $\mathrm{Eu} 1-\mathrm{N} 3$ & $2.479(3)$ \\
$\mathrm{C} 1-\mathrm{N} 1$ & $1.301(3)$ & $\mathrm{C} 1-\mathrm{N} 1$ & $1.307(4)$ \\
& & $\mathrm{Eu} 1-\mathrm{O} 1$ & $2.635(3)$ \\
$\mathrm{N} 2-\mathrm{Yb} 1-\mathrm{C} 1$ & $119.22(7)$ & $\mathrm{N} 2-\mathrm{Eu} 1-\mathrm{C} 1$ & $131.98(9)$ \\
$\mathrm{C} 1-\mathrm{Yb} 1-\mathrm{N} 3$ & $113.34(7)$ & $\mathrm{C} 1-\mathrm{Eu} 1-\mathrm{N} 3$ & $102.68(9)$ \\
$\mathrm{N} 2-\mathrm{Yb} 1-\mathrm{N} 3$ & $116.97(8)$ & $\mathrm{N} 2-\mathrm{Eu} 1-\mathrm{N} 3$ & $107.46(9)$ \\
& & $\mathrm{O} 1-\mathrm{Eu} 1-\mathrm{C} 1$ & $95.32(9)$ \\
\hline
\end{tabular}




\section{DFT Calculations}

All calculations were performed with the Gaussian 09 suite of programs, revision E. 01. ${ }^{\text {S14 }}$ The geometry of 2 was optimized with Density Functional Theory (DFT) method with PBE1PBE functional. Ytterbium was treated with a large-core Stuttgart Dresden relativistic effective core potential (RECP). ${ }^{515}$ Hydrogen, carbon, nitrogen and silicon atoms were described with a 6-31G(d,p) basis set. NBO/NLMO analysis was performed using NBO 6.0. ${ }^{\mathrm{S} 16}$

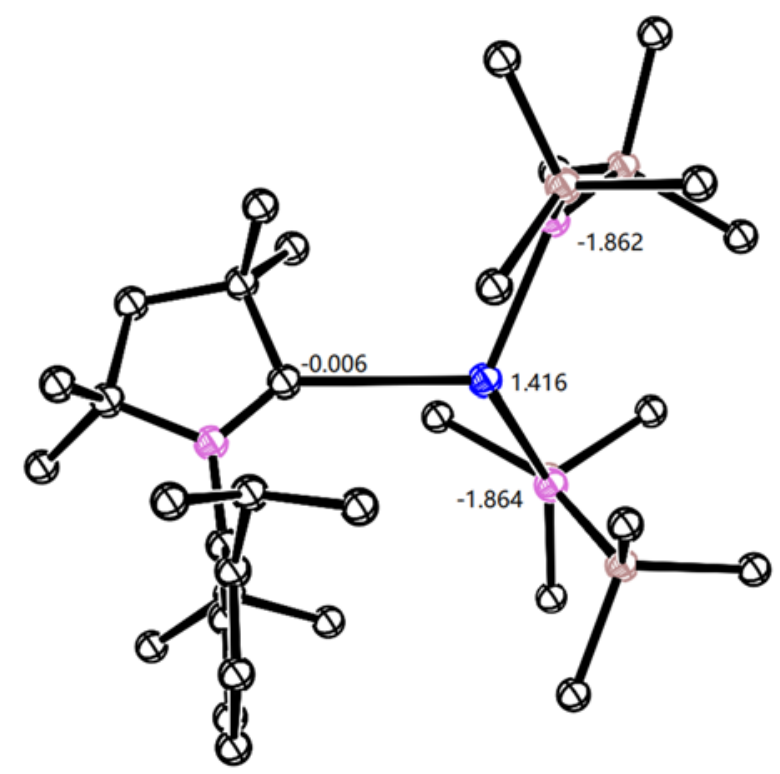

Figure S1. Computationally optimized structures of complex 2. Hydrogen atoms are omitted for clarity. Natural charges from the NBO analysis are shown along respective atoms. Color code: black, carbon; purple, nitrogen; brown, silicon; blue, ytterbium.

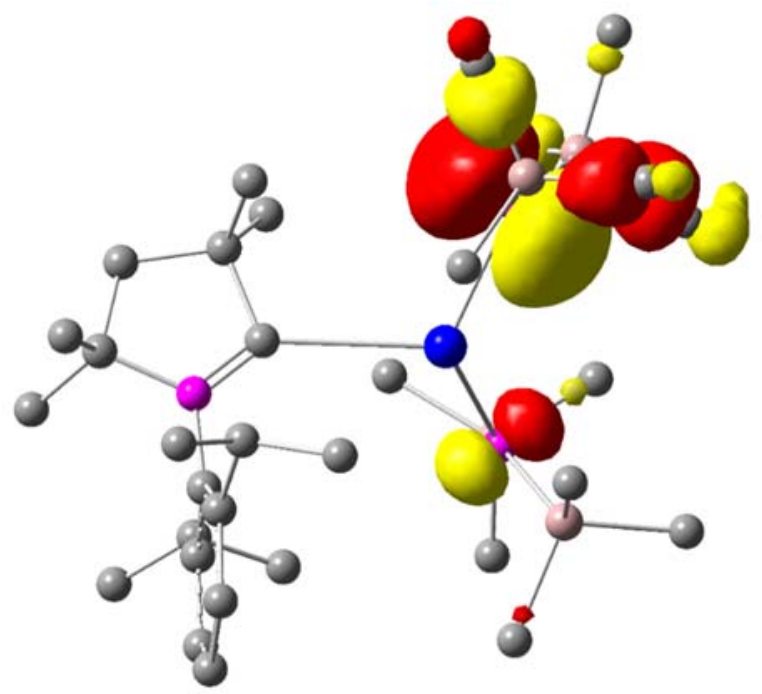

Figure S2. DFT-computed HOMO (based on the silylamide ligands) for complex 2. 
Table S4. Comparison of experimental and calculated metrical parameters in 2.

\begin{tabular}{ccc}
\hline & Experimental & Calculated (PBE1PBE) \\
\hline Yb1-N2 & $2.321(2)$ & 2.355 \\
Yb1-N3 & $2.327(2)$ & 2.363 \\
Yb1-C1 & $2.672(3)$ & 2.713 \\
C1-N1 & $1.301(3)$ & 1.306 \\
C1-C2 & $1.528(3)$ & 1.516 \\
N2-Si1 & $1.693(2)$ & 1.714 \\
N2-Si2 & $1.685(2)$ & 1.703 \\
N3-Si3 & $1.695(2)$ & 1.718 \\
N3-Si4 & $1.691(2)$ & 1.704 \\
N2-Yb1-C1 & $119.22(7)$ & 114.9 \\
C1-Yb1-N3 & $113.34(7)$ & 112.6 \\
N2-Yb1-N3 & $116.97(8)$ & 119.6 \\
N1-C1-Yb1 & $141.45(18)$ & 140.0 \\
C2-C1-Yb1 & $111.33(16)$ & 112.0 \\
C2-C1-N1 & $107.2(2)$ & 107.9 \\
Yb1-N3-Si3 & $123.20(11)$ & 122.1 \\
Yb1-N3-Si4 & $111.96(11)$ & 111.3 \\
Si3-N3-Si4 & $123.04(13)$ & 122.2 \\
Si1-N2-Si2 & $123.23(13)$ & 123.8 \\
Yb1-N2-Si1 & $132.74(12)$ & 131.6 \\
Yb1-N2-Si2 & $103.85(10)$ & 103.9 \\
\hline
\end{tabular}




\section{Cartesian Coordinates of the Optimized Structures}

Complex 2

\begin{tabular}{|c|c|c|c|}
\hline $\mathrm{Yb}$ & -0.79845100 & -0.10077500 & 0.20256400 \\
\hline $\mathrm{Si}$ & -4.06400100 & -0.99173200 & -0.96140500 \\
\hline $\mathrm{Si}$ & -1.52907700 & 3.33737300 & -0.99037900 \\
\hline $\mathrm{Si}$ & -2.63467100 & -2.89987700 & 0.86278100 \\
\hline $\mathrm{Si}$ & -1.33689600 & 2.58318100 & 1.92352300 \\
\hline $\mathrm{N}$ & 2.79582400 & -0.83646000 & -0.83443700 \\
\hline $\mathrm{N}$ & -1.22302500 & 2.20928200 & 0.26333800 \\
\hline $\mathrm{N}$ & -2.64817600 & -1.52438100 & -0.14583700 \\
\hline $\mathrm{C}$ & 1.50206800 & -0.94166400 & -0.96801400 \\
\hline $\mathrm{C}$ & 2.58815500 & -1.88409900 & -2.92265000 \\
\hline $\mathrm{H}$ & 2.74944800 & -2.87717500 & -3.35357300 \\
\hline $\mathrm{H}$ & 2.64225000 & -1.16021100 & -3.74378400 \\
\hline $\mathrm{C}$ & 3.72502300 & -0.79116900 & 1.44891700 \\
\hline $\mathrm{C}$ & 3.65102200 & -1.54476700 & -1.87400700 \\
\hline $\mathrm{C}$ & 3.32524800 & 2.05191300 & -1.13794600 \\
\hline $\mathrm{H}$ & 2.83839200 & 1.38535100 & -1.85859300 \\
\hline $\mathrm{C}$ & 1.22568200 & -1.75951500 & -2.21171300 \\
\hline $\mathrm{C}$ & 4.46627700 & -0.10621100 & 2.41420500 \\
\hline $\mathrm{H}$ & 4.70496800 & -0.60651300 & 3.34624400 \\
\hline $\mathrm{C}$ & 4.50960300 & 1.86556300 & 1.05771900 \\
\hline $\mathrm{H}$ & 4.77970900 & 2.90772000 & 0.93099600 \\
\hline $\mathrm{C}$ & 4.74299500 & -0.63982500 & -2.42159700 \\
\hline $\mathrm{H}$ & 4.33391000 & 0.23803300 & -2.92534500 \\
\hline $\mathrm{H}$ & 5.32373700 & -1.20251100 & -3.15926200 \\
\hline $\mathrm{H}$ & 5.43197900 & -0.30797900 & -1.63903500 \\
\hline $\mathrm{C}$ & 0.18945600 & -1.05146300 & -3.08528300 \\
\hline $\mathrm{H}$ & -0.79482900 & -1.01281700 & -2.60307300 \\
\hline $\mathrm{H}$ & 0.04846200 & -1.60319000 & -4.02150200 \\
\hline $\mathrm{H}$ & 0.49314900 & -0.03046900 & -3.33994600 \\
\hline $\mathrm{C}$ & 3.43702900 & -0.12340200 & 0.24373700 \\
\hline $\mathrm{C}$ & 4.87823100 & 1.20125000 & 2.21689100 \\
\hline $\mathrm{H}$ & 5.45495600 & 1.71323700 & 2.97947000 \\
\hline $\mathrm{C}$ & 3.78109200 & 1.22848500 & 0.05165300 \\
\hline $\mathrm{C}$ & 0.64883400 & -3.11295600 & -1.77066000 \\
\hline $\mathrm{H}$ & 1.30299200 & -3.64652500 & -1.07293200 \\
\hline $\mathrm{H}$ & 0.50329600 & -3.75241400 & -2.64828800 \\
\hline $\mathrm{H}$ & -0.32750100 & -2.98461100 & -1.29214600 \\
\hline $\mathrm{C}$ & 4.47799800 & 2.76213600 & -1.85016300 \\
\hline $\mathrm{H}$ & 4.90279900 & 3.55864500 & -1.23058500 \\
\hline $\mathrm{H}$ & 4.11499500 & 3.23551300 & -2.76847800 \\
\hline $\mathrm{H}$ & 5.29393700 & 2.08416500 & -2.11601400 \\
\hline $\mathrm{C}$ & 2.28193800 & 3.07698600 & -0.68776800 \\
\hline
\end{tabular}




\begin{tabular}{|c|c|c|c|}
\hline $\mathrm{H}$ & 1.38163400 & 2.61526300 & -0.27019900 \\
\hline $\mathrm{H}$ & 1.97149100 & 3.69822400 & -1.53388200 \\
\hline $\mathrm{H}$ & 2.69420700 & 3.74614700 & 0.07595100 \\
\hline $\mathrm{C}$ & -0.75754100 & 2.72502700 & -2.61916200 \\
\hline $\mathrm{H}$ & 0.31983400 & 2.55096100 & -2.52144400 \\
\hline $\mathrm{H}$ & -0.90082700 & 3.46019500 & -3.41984700 \\
\hline $\mathrm{H}$ & -1.21400700 & 1.79206400 & -2.96860100 \\
\hline $\mathrm{C}$ & 4.29592000 & -2.77953300 & -1.25599900 \\
\hline $\mathrm{H}$ & 4.99026400 & -2.49990500 & -0.45991900 \\
\hline $\mathrm{H}$ & 4.87060800 & -3.30397200 & -2.02535100 \\
\hline $\mathrm{H}$ & 3.56205900 & -3.48069600 & -0.85130400 \\
\hline $\mathrm{C}$ & -0.96276800 & -2.92285900 & 1.79095000 \\
\hline $\mathrm{H}$ & -0.08961800 & -2.88773300 & 1.12345200 \\
\hline $\mathrm{H}$ & -0.86038300 & -3.84654400 & 2.37190500 \\
\hline $\mathrm{H}$ & -0.87577100 & -2.10455700 & 2.51886000 \\
\hline $\mathrm{C}$ & 3.15913500 & -2.15147400 & 1.81208000 \\
\hline $\mathrm{H}$ & 2.63049900 & -2.55502900 & 0.94235100 \\
\hline $\mathrm{C}$ & -3.59611400 & -0.12731000 & -2.58298400 \\
\hline $\mathrm{H}$ & -2.90115500 & 0.70276600 & -2.41686500 \\
\hline $\mathrm{H}$ & -4.47745400 & 0.29818100 & -3.07716400 \\
\hline $\mathrm{H}$ & -3.12668800 & -0.82191600 & -3.29001500 \\
\hline $\mathrm{C}$ & -2.75414300 & -4.57301800 & -0.02781300 \\
\hline $\mathrm{H}$ & -3.75582400 & -4.73582500 & -0.43897100 \\
\hline $\mathrm{H}$ & -2.53896700 & -5.40660900 & 0.65250500 \\
\hline $\mathrm{H}$ & -2.04853800 & -4.63690900 & -0.86434900 \\
\hline $\mathrm{C}$ & -5.27091400 & -2.37866700 & -1.43905500 \\
\hline $\mathrm{H}$ & -4.79528100 & -3.12141400 & -2.08959200 \\
\hline $\mathrm{H}$ & -6.13296700 & -1.96903900 & -1.97925100 \\
\hline $\mathrm{H}$ & -5.66292100 & -2.91174600 & -0.56480900 \\
\hline $\mathrm{C}$ & -5.03143500 & 0.26660800 & 0.07696100 \\
\hline $\mathrm{H}$ & -5.38995700 & -0.18672000 & 1.00905800 \\
\hline $\mathrm{H}$ & -5.90285400 & 0.67055300 & -0.45333600 \\
\hline $\mathrm{H}$ & -4.38913400 & 1.11305200 & 0.34830600 \\
\hline $\mathrm{C}$ & -0.82149400 & 5.06881600 & -0.65174600 \\
\hline $\mathrm{H}$ & -1.28872000 & 5.52190100 & 0.23127200 \\
\hline $\mathrm{H}$ & -1.00944600 & 5.74439000 & -1.49521400 \\
\hline $\mathrm{H}$ & 0.25880300 & 5.05143000 & -0.47225800 \\
\hline $\mathrm{C}$ & -3.95462700 & -2.89675500 & 2.22757100 \\
\hline $\mathrm{H}$ & -3.83195400 & -2.03330000 & 2.89285700 \\
\hline $\mathrm{H}$ & -3.90906900 & -3.80211600 & 2.84559200 \\
\hline $\mathrm{H}$ & -4.96642500 & -2.83720700 & 1.81098100 \\
\hline $\mathrm{C}$ & 2.12731200 & -1.97953200 & 2.93034100 \\
\hline 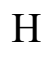 & 2.60427300 & -1.64398800 & 3.85724300 \\
\hline $\mathrm{H}$ & 1.60961400 & -2.92096000 & 3.13627800 \\
\hline
\end{tabular}




$\begin{array}{lrrr}\mathrm{H} & 1.37105000 & -1.23401600 & 2.66929000 \\ \mathrm{C} & -3.37526000 & 3.59647400 & -1.33529400 \\ \mathrm{H} & -3.85928500 & 2.67991400 & -1.68832200 \\ \mathrm{H} & -3.53595900 & 4.37122000 & -2.09550400 \\ \mathrm{H} & -3.90629300 & 3.90797400 & -0.42879900 \\ \mathrm{C} & 4.21767200 & -3.16636800 & 2.24513000 \\ \mathrm{H} & 5.01427400 & -3.29476600 & 1.50803100 \\ \mathrm{H} & 3.75371900 & -4.14437400 & 2.41053400 \\ \mathrm{H} & 4.68968600 & -2.87273700 & 3.18864700 \\ \mathrm{C} & -2.96694800 & 3.37669000 & 2.46467600 \\ \mathrm{H} & -3.11802500 & 4.34413100 & 1.97148800 \\ \mathrm{H} & -3.00230900 & 3.55350800 & 3.54660800 \\ \mathrm{H} & -3.81959400 & 2.74124200 & 2.19894600 \\ \mathrm{C} & 0.08247400 & 3.63343300 & 2.61537400 \\ \mathrm{H} & 1.05542800 & 3.17487200 & 2.40041600 \\ \mathrm{H} & 0.00436900 & 3.75374800 & 3.70308400 \\ \mathrm{H} & 0.09374100 & 4.63472600 & 2.17208200 \\ \mathrm{C} & -1.22434100 & 0.92993300 & 2.91073300 \\ \mathrm{H} & -2.00099900 & 0.19298000 & 2.64802200 \\ \mathrm{H} & -1.39029200 & 1.14199400 & 3.97171700 \\ \mathrm{H} & -0.23274500 & 0.44594400 & 2.88062400\end{array}$




\section{References}

[S1] Hitchcock, P. B.; Khvostov, A. V.; Lappert, M. F.; Protchenko, A. V. Ytterbium(II) amides and crown ethers: addition versus amide substitution. J. Organomet. Chem. 2002, 647, 198-204.

[S2] (a) Evans, W. J.; Drummond, D. K.; Zhang, H. M.; Atwood, J. L. Synthesis and X-ray crystal structure of the divalent [bis(trimethylsilyl)amido] samarium complexes $\left[\left(\mathrm{Me}_{3} \mathrm{Si}\right)_{2} \mathrm{~N}\right]_{2} \mathrm{Sm}(\mathrm{THF})_{2}$ and $\left\{\left[\left(\mathrm{Me}_{3} \mathrm{Si}\right)_{2} \mathrm{~N}\right] \mathrm{Sm}(\mu-\mathrm{I})(\mathrm{DME})(\mathrm{THF})\right\}_{2}$. Inorg. Chem. 1988, 27, 575-579. (b) Bienfait, A. M.; Wolf, B. M.; Törnroos, K. W.; Anwander, R. Trivalent Rare-Earth-Metal Bis(trimethylsilyl)amide Halide Complexes by Targeted Oxidations. Inorg. Chem. 2018, 57, 5204-5212.

[S3] (a) Jazzar, R.; Dewhurst, R. D.; Bourg, J. B.; Donnadieu, B.; Canac, Y.; Bertrand, G. Intramolecular "hydroiminiumation" of alkenes: application to the synthesis of conjugate acids of cyclic alkyl amino carbenes (CAACs). Angew. Chem. Int. Ed. 2007, 46, 2899-2902. (b) Müller, C.; Andrada, D. M.; Bischoff, I. A.; Zimmer, M.; Huch, V.; Steinbrück, N.; Schafer, A. Synthesis, Structure, and Bonding Analysis of Tin(II) Dihalide and Cyclopentadienyltin(II) Halide (Alkyl) (amino)carbene Complexes. Organometallics 2019, 38, 1052-1061. (c) Bissinger, P.; Braunschweig, H.; Damme, A.; Krummenacher, I.; Phukan, A. K.; Radacki, K.; Sugawara, S. Isolation of a Neutral Boron-Containing Radical Stabilized by a Cyclic (Alkyl)(Amino)Carbene. Angew. Chem. Int. Ed. 2014, 53, 7360-7363.

[S4] Fu, P.-F.; Brard, L.; Li, Y.; Marks, T. Regioselection and Enantioselection in OrganolanthanideCatalyzed Olefin Hydrosilylation. A Kinetic and Mechanistic Study. J. Am. Chem. Soc. 1995, 117, $7157-7168$.

[S5] Hu, M.; He, Q.; Fan, S.; Wang, Z.; Liu, L.; Mu, Y.; Peng, Q.; Zhu, S. Ligands with 1,10phenanthroline scaffold for highly regioselective iron-catalyzed alkene hydrosilylation. Nat. Commun. 2018, 9, 1-11.

[S6] Junquera, L. B.; Puerta, M. C.; Valerga, P. R-Allyl nickel(II) complexes with chelating Nheterocyclic carbenes: synthesis, structural characterization, and catalytic activity. Organometallics 2012, 31, 2175-2183.

[S7] Wang, C.; Teo, W. J.; Ge, S. Cobalt-Catalyzed Regiodivergent Hydrosilylation of Vinylarenes and Aliphatic Alkenes: Ligand- and Silane-Dependent Regioselectivities. ACS Catal. 2017, 7, $855-863$. 
[S8] Zaranek, M.; Witomska, S.; Patroniak, V.; Pawluc, P. Unexpected catalytic activity of simple triethylborohydrides in the hydrosilylation of alkenes. Chem. Commun. 2017, 53, 5404-5407.

[S9] Dong, Y.; Xie, S.; Zhang, P.; Fan, Q.; Du, X.; Sun, H.; Li, X.; Fuhr, O.; Fenske, D. Selectivity Reverse of Hydrosilylation of Aryl Alkenes Realized by Pyridine N-Oxide with [PSiP] Pincer Cobalt(III) Hydride as Catalyst. Inorg. Chem. 2021, 60, 4551-4562.

[S10] (a) Jr, M. W. G.; Pirnot, M. T.; Bandar, J. S.; Liu, R. Y.; Buchwald, S. L. Asymmetric Copper Hydride-Catalyzed Markovnikov Hydrosilylation of Vinylarenes and Vinyl Heterocycles. J. Am. Chem. Soc. 2017, 139, 2192-2195. (b) Zuo, Z.; Xu, S.; Zhang, L.; Gan, L.; Fang, H.; Liu, G.; Huang, Z. Cobalt-Catalyzed Asymmetric Hydrogenation of Vinylsilanes with a PhosphinePyridine-Oxazoline Ligand: Synthesis of Optically Active Organosilanes and Silacycles. Organometallics 2019, 38, 3906-3911.

[S11] Zhang, S.; Liao, J.; Unruh, D. K.; Li, G.; Findlater, M. Cobalt- and iron-catalyzed regiodivergent alkene hydrosilylations. Org. Chem. Front. 2021, 8, 2174-2181.

[S12] Sheldrick, G. M. A short history of SHELX. Acta Crystallogr. 2008, A64, 112-122.

[S13] Farrugia, L. J. ORTEP-3 for windows - a version of ORTEP-III with a graphical user interface (GUI). J. Appl. Crystallogr. 1997, 30, 565.

[S14] Frisch, M. J.; Trucks, G. W.; Schlegel, H. B.; Scuseria, G. E.; Robb, M. A.; Cheeseman, J. R.; Scalmani, G.; Barone, V.; Mennucci, B.; Petersson, G. A.; Nakatsuji, H.; Caricato, M.; Li, X.; Hratchian, H. P.; Izmaylov, A. F.; Bloino, J.; Zheng, G.; Sonnenberg, J. L.; Hada, M.; Ehara, M.; Toyota, K.; Fukuda, R.; Hasegawa, J.; Ishida, M.; Nakajima, T.; Honda, Y.; Kitao, O.; Nakai, H.; Vreven, T.; Montgomery, J. A.; Jr.; Peralta, J. E.; Ogliaro, F.; Bearpark, M.; Heyd, J. J.; Brothers, E.; Kudin, K. N.; Staroverov,V. N.; Keith, T.; Kobayashi, R.; Normand, J.; Raghavachari, K.; Rendell, A.; Burant, J. C.; Iyengar, S. S.; Tomasi, J.; Cossi, M.; Rega, N.; Millam, J. M.; Klene, M.; Knox, J. E.; Cross, J. B.; Bakken, V.; Adamo, C.; Jaramillo, J.; Gomperts, R.; Stratmann, R. E.; Yazyev, O.; Austin, A. J.; Cammi, R.; Pomelli, C.; Ochterski, J. W.; Martin, R. L.; Morokuma, K.; Zakrzewski, V. G.; Voth, G. A.; Salvador, P. J.; Dannenberg, J.; Dapprich, S.; Daniels, A. D.; Farkas, O.; Foresman, J. B.; Ortiz, J. V.; Cioslowski, J.; Fox, D. J. Gaussian 09, Revision E. 01, Gaussian, Inc., Wallingford CT, 2013.

[S15] Dolg, M.; Stoll, H.; Savin, A.; Preuss, H. Energy-adjusted pseudopotentials for the rare earth elements. Theor. Chim. Acta. 1989, 75, 173-194. 
[S16] Glendening, E. D.; Badenhoop, J. K.; Reed, A. E.; Carpenter, J. E.; Bohmann, J. A.; Morales, C. M.; Landis, C. R.; Weinhold, F. NBO 6.0; Theoretical Chemistry Institute, University of Wisconsin: Madison,WI, 2013. 


\section{GC-MS Spectra of Crude Reaction Mixtures}

Rा: 8.26- 19.04<smiles>CC(c1ccccc1)c1ccc(N(C)[14CH3])cc1</smiles>

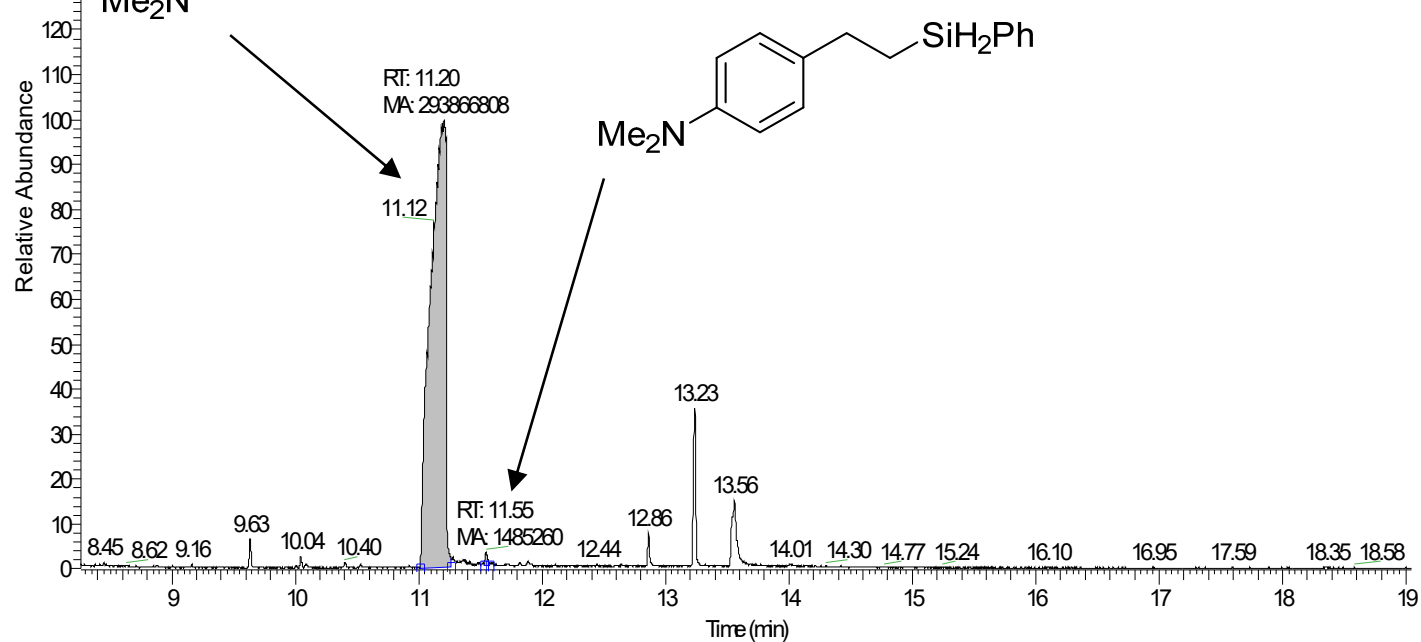

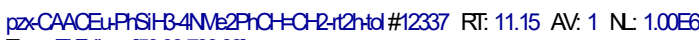
T: +cEFul ms [50.00-700.00]

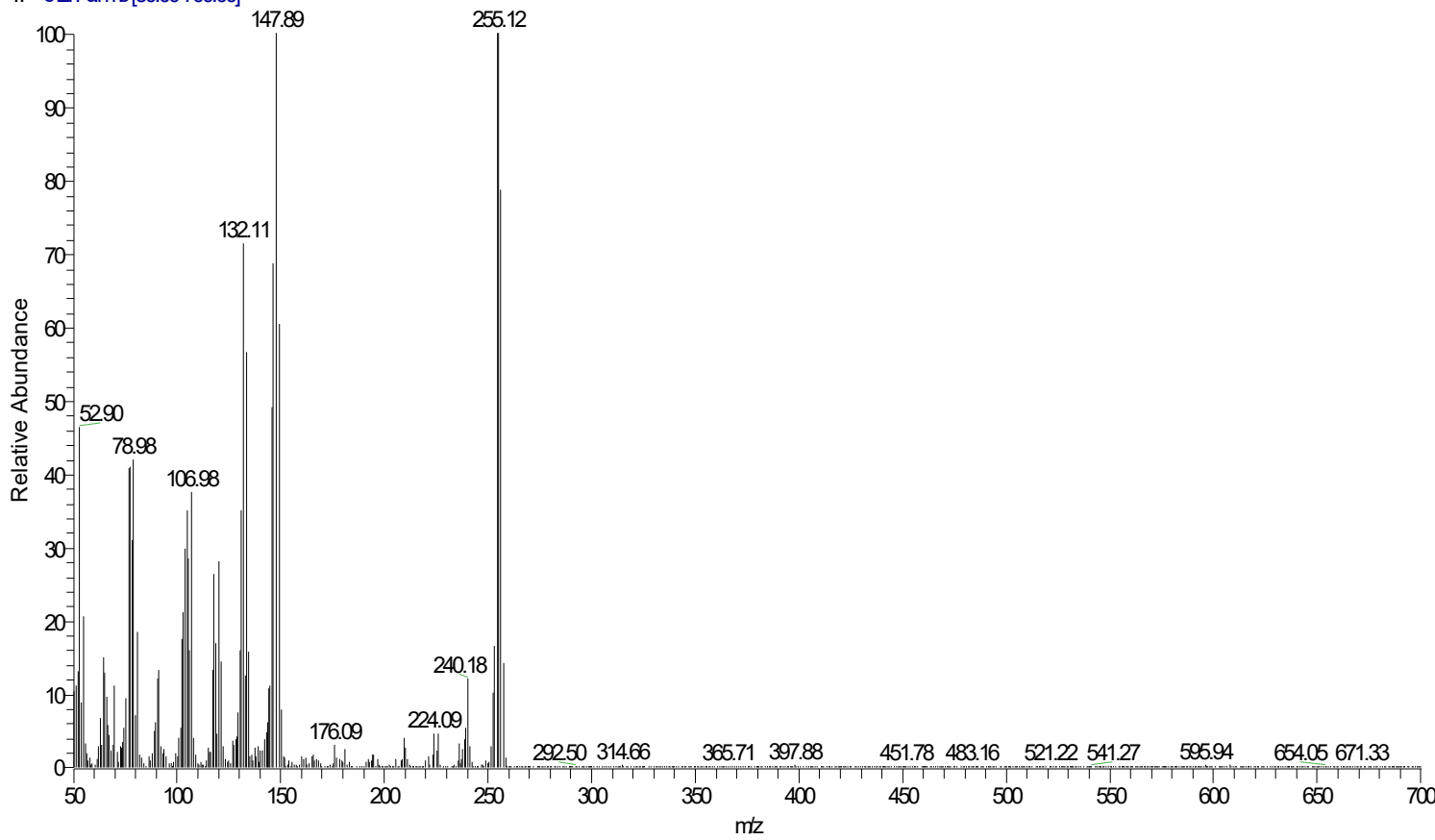

Figure S3. GC-MS spectrum of crude reaction mixture from $4 \mathbf{i}$ and $\mathrm{PhSiH}_{3}$. 


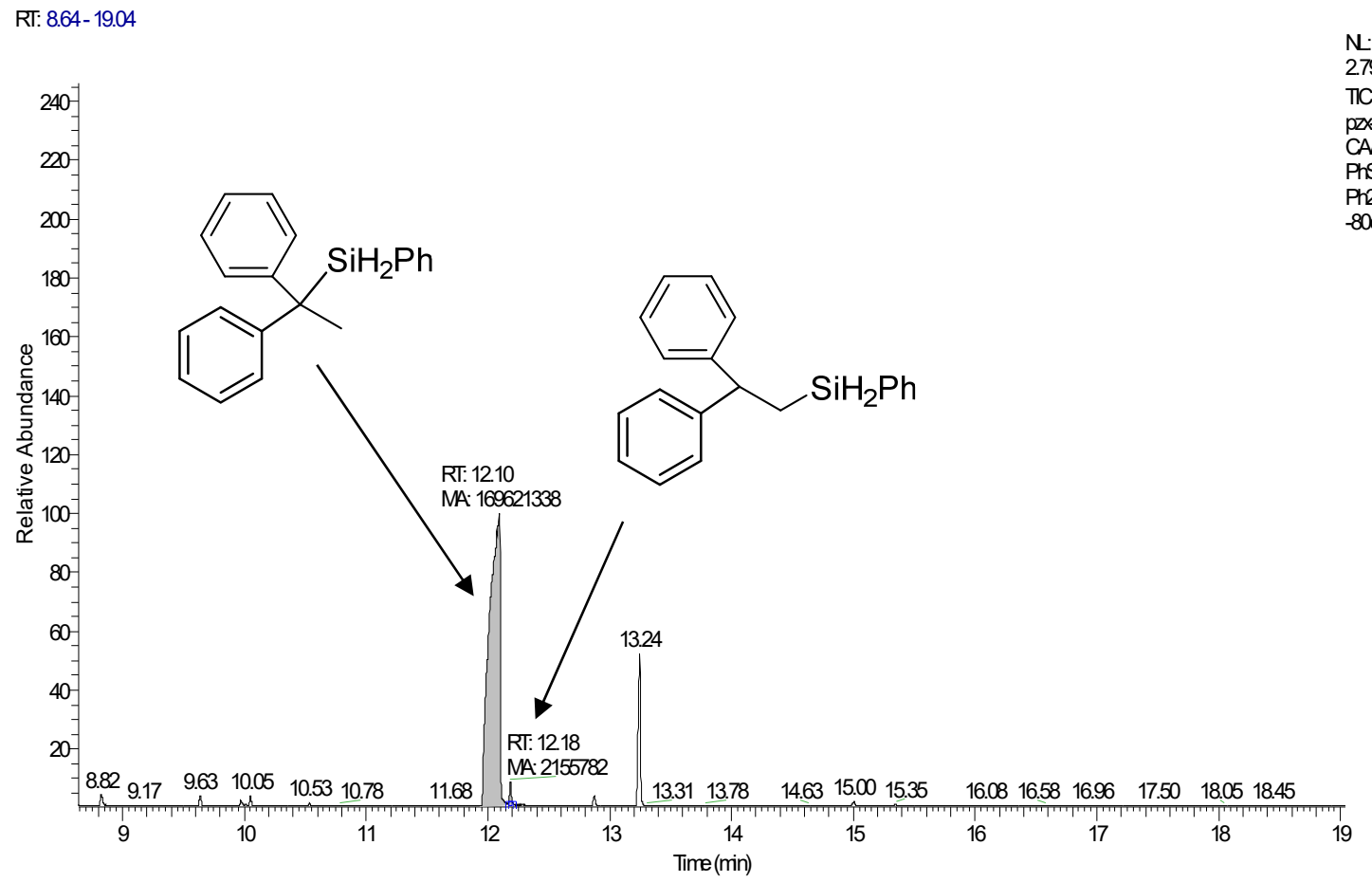

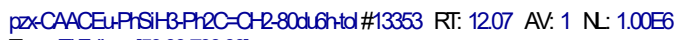

T: + cEF Full ms [50.00-700.00]

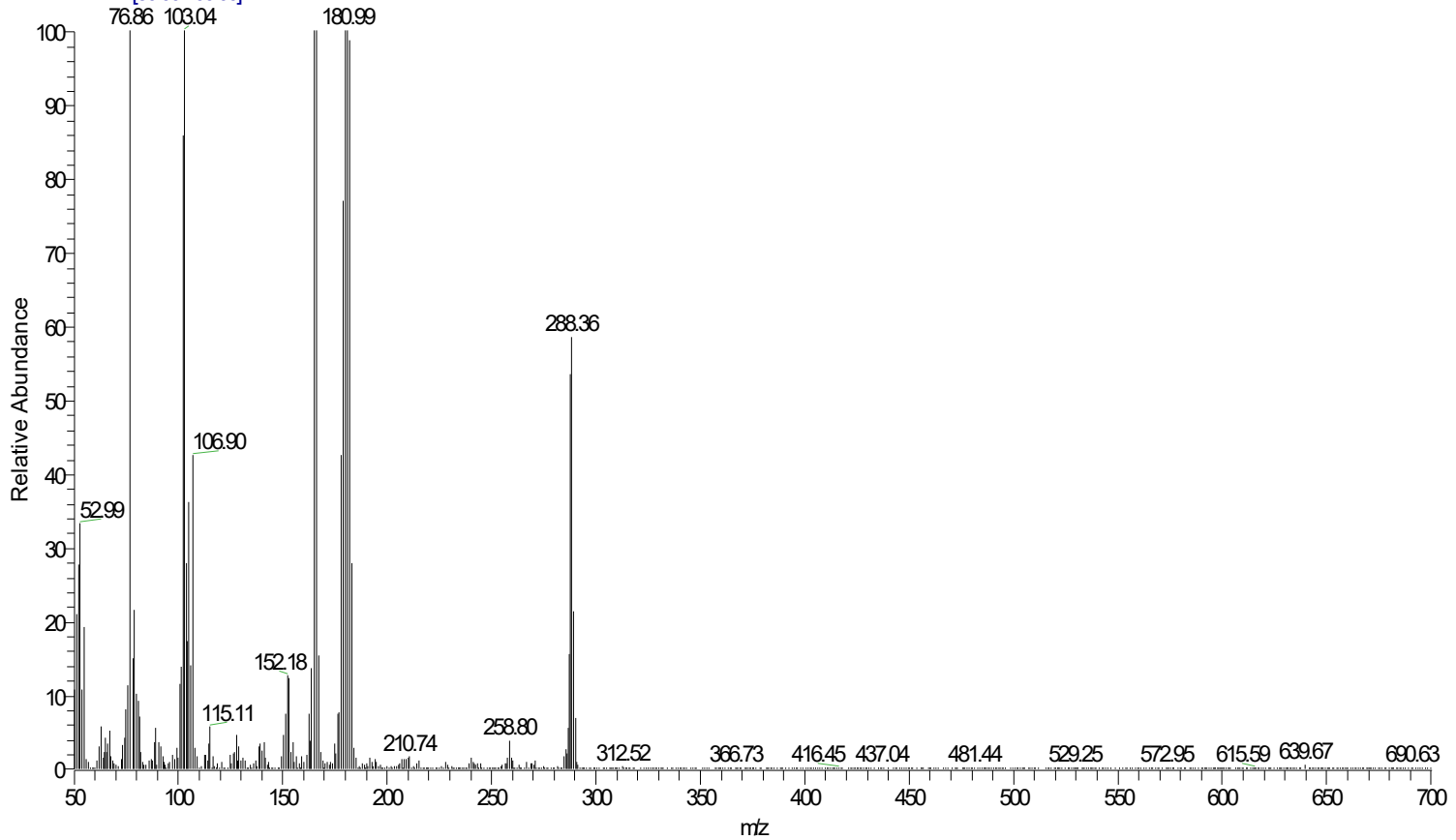

Figure S4. GC-MS spectrum of crude reaction mixture from $4 \mathbf{l}$ and $\mathrm{PhSiH}_{3}$. 


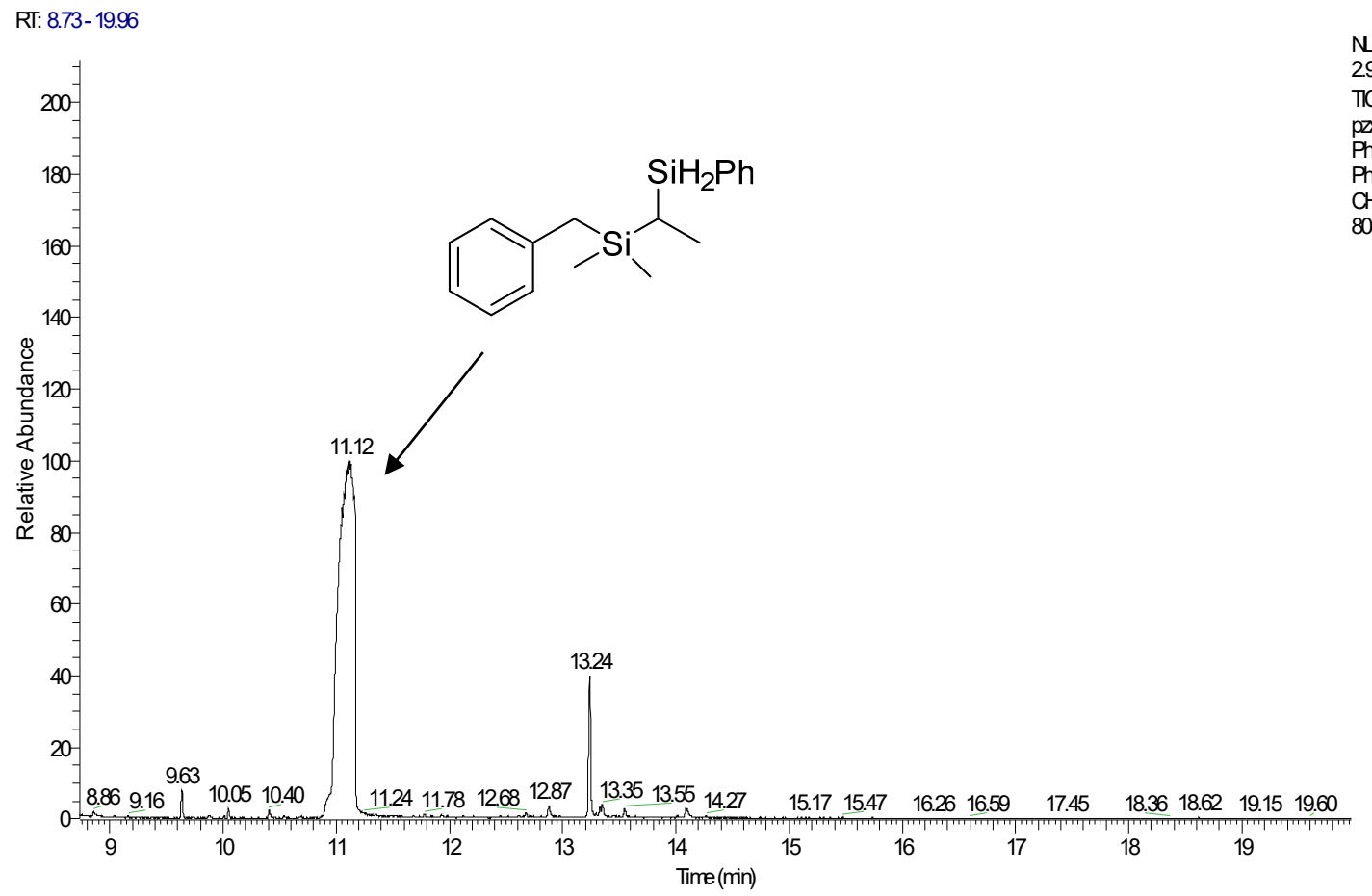

pZX-CAACEUPhSiHBPhCH-ME2SICHFC-R-80duGh-td\#12168 RT: 11.00 AV: 1 N-1.00E6

T: +cEFFul ms [50.00-700.00]

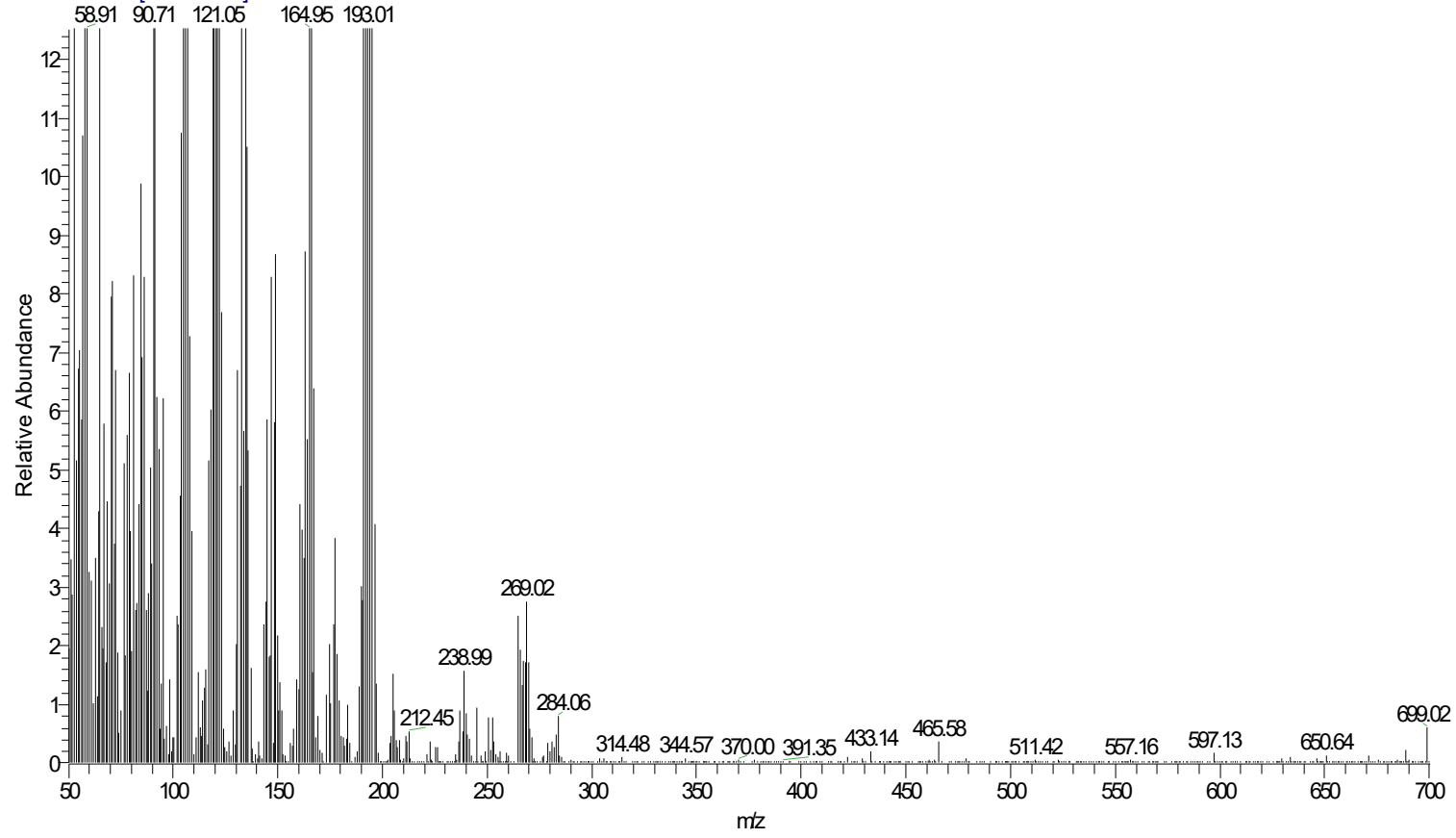

Figure S5. GC-MS spectrum of crude reaction mixture from $4 p$ and $\mathrm{PhSiH}_{3}$. 


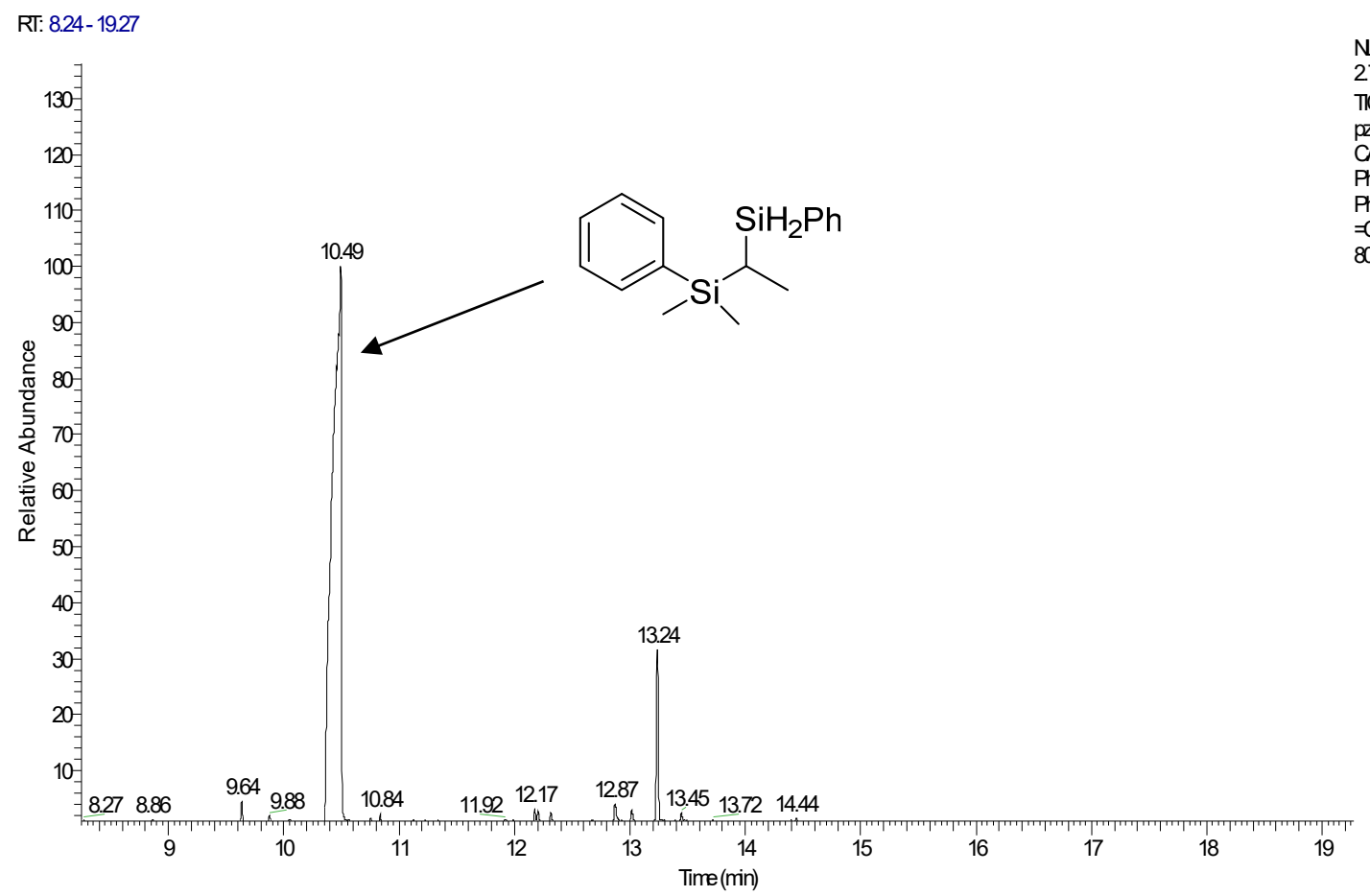

pZX-CAACEU PhSiHBPHM2SiCH=CH-80dubhtol \#11585 RT: 10.47 AV: 1 N-1.00E6

T: +cEFful ms [50.00-700.00]

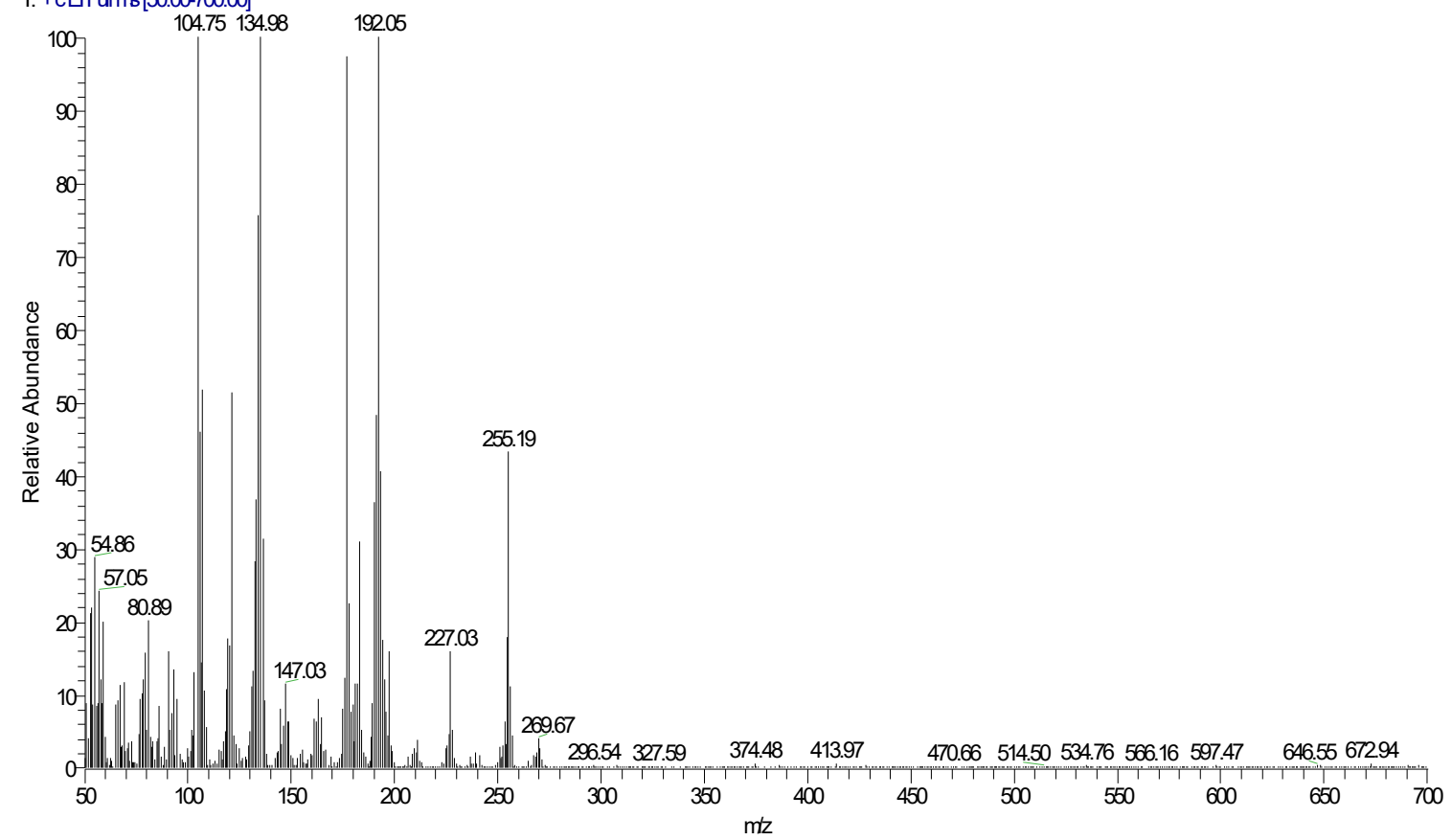

Figure S6. GC-MS spectrum of crude reaction mixture from $\mathbf{4 q}$ and $\mathrm{PhSiH}_{3}$. 


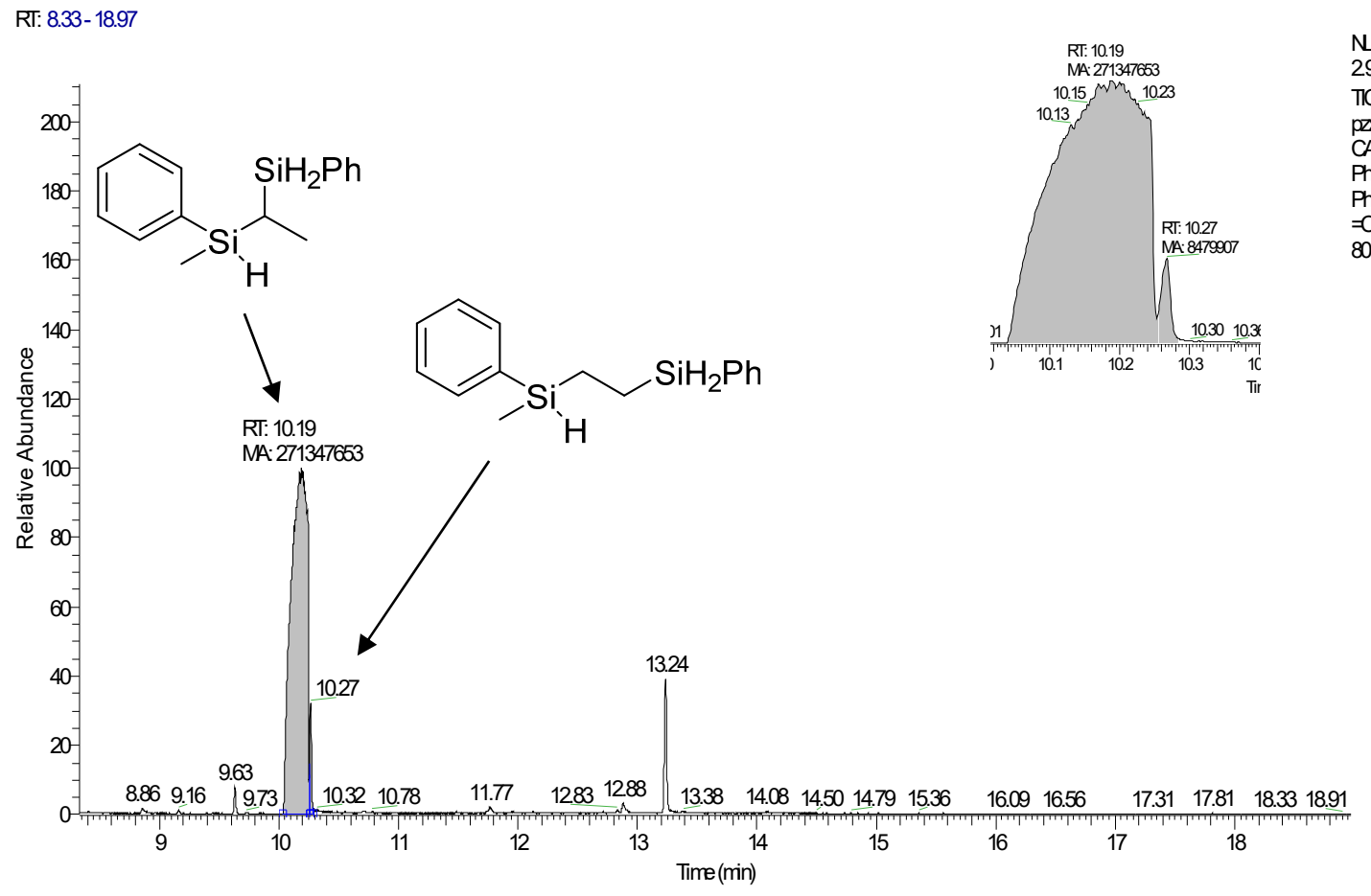

$\mathrm{N}$ : TIC MS CAACER

PhSiH$\mathrm{PHMSHCH}$ $=\mathrm{CH}-$ BOduAhtol

pZX-CAACEU PhSiHBPMMSSHCHFCHR-80duAhtd \#11274 RT: 10.19 AV: 1 N- 1.00E6 T: + cEFFull ms [50.00-700.00]

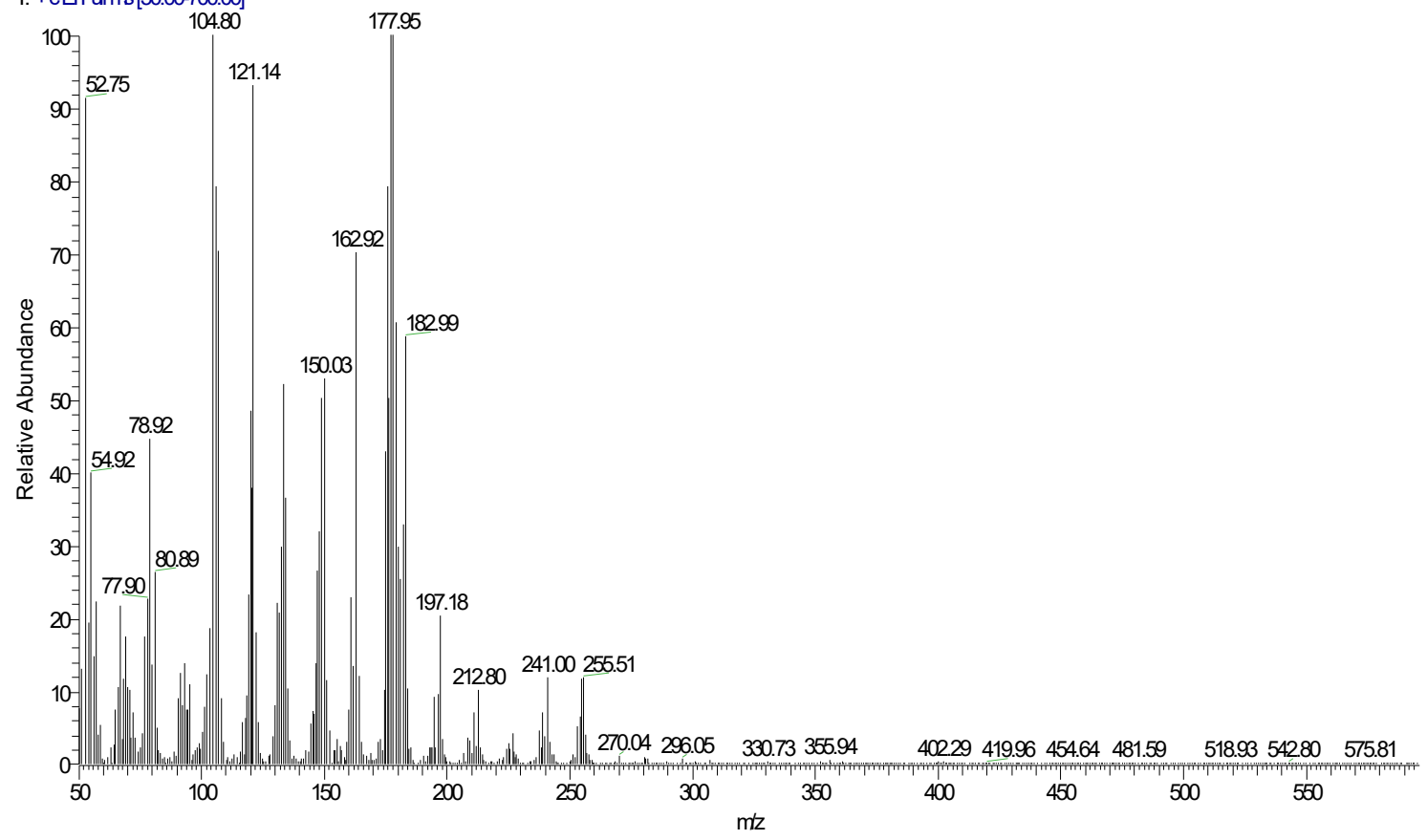

Figure S7. GC-MS spectrum of crude reaction mixture from $4 \mathbf{s}$ and $\mathrm{PhSiH}_{3}$. 

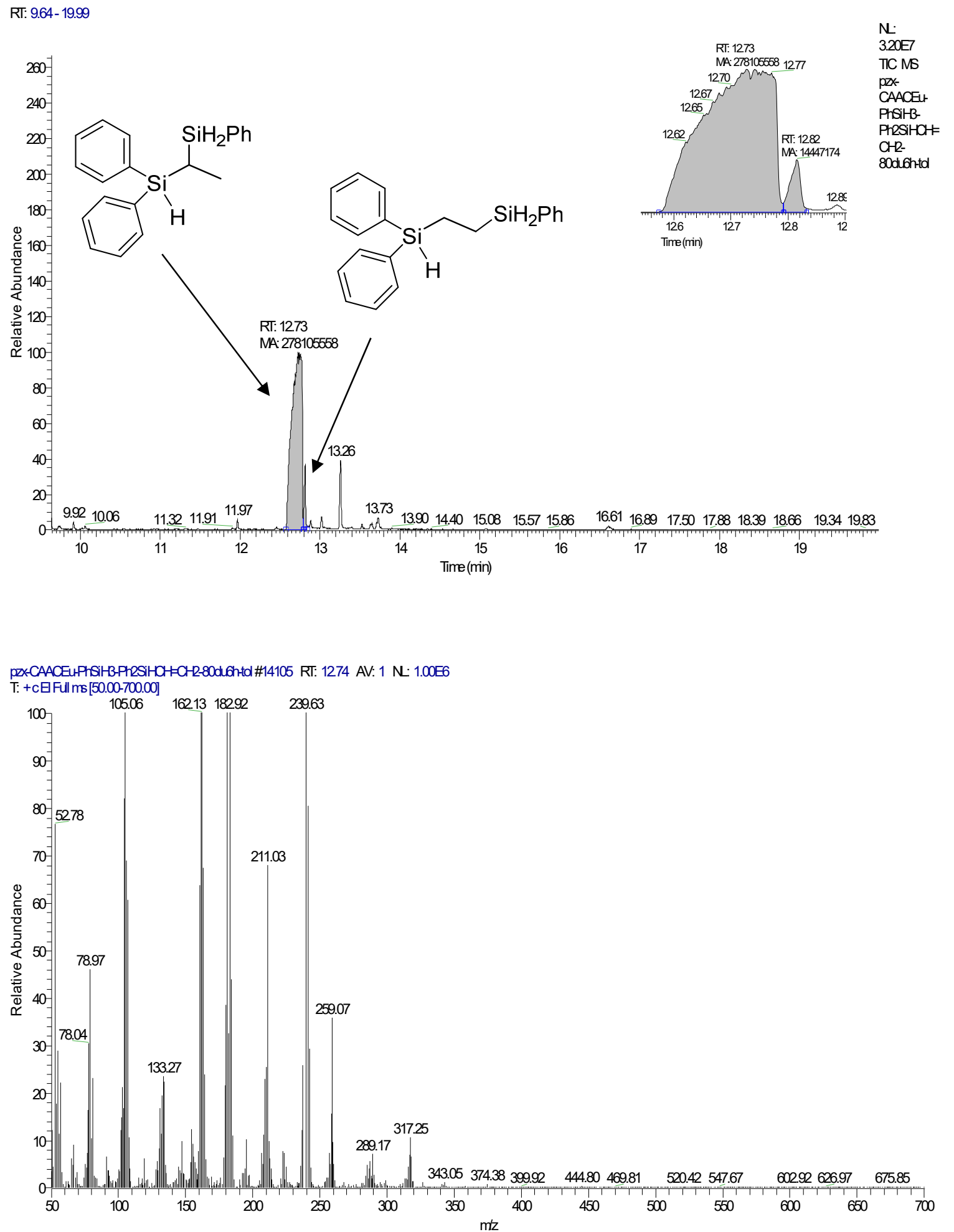

Figure S8. GC-MS spectrum of crude reaction mixture from $4 \mathbf{t}$ and $\mathrm{PhSiH}_{3}$. 


\section{NMR Spectra}

紐总易

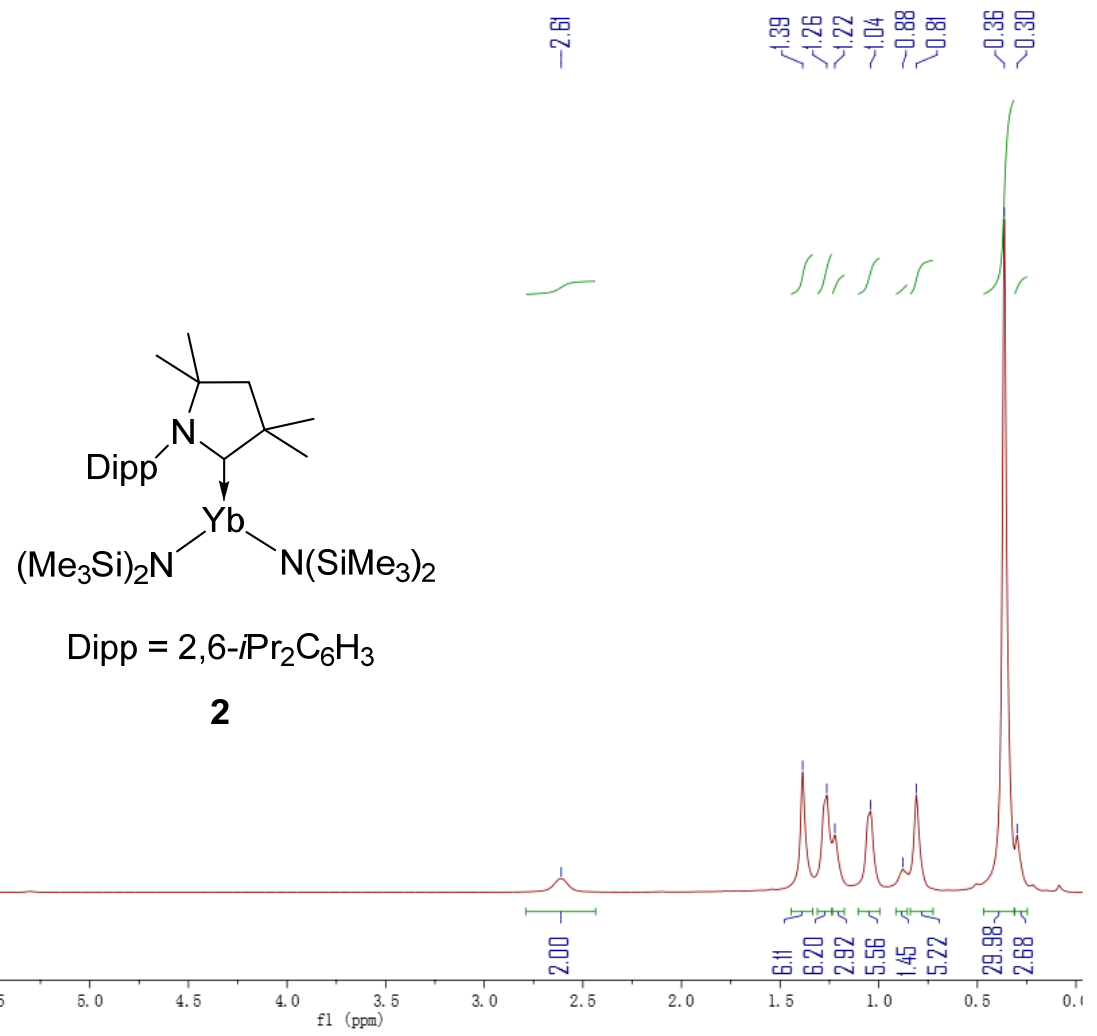

Figure S9. ${ }^{1} \mathrm{H}$ NMR spectrum of 2 in $\mathrm{C}_{6} \mathrm{D}_{6}(298 \mathrm{~K})$.

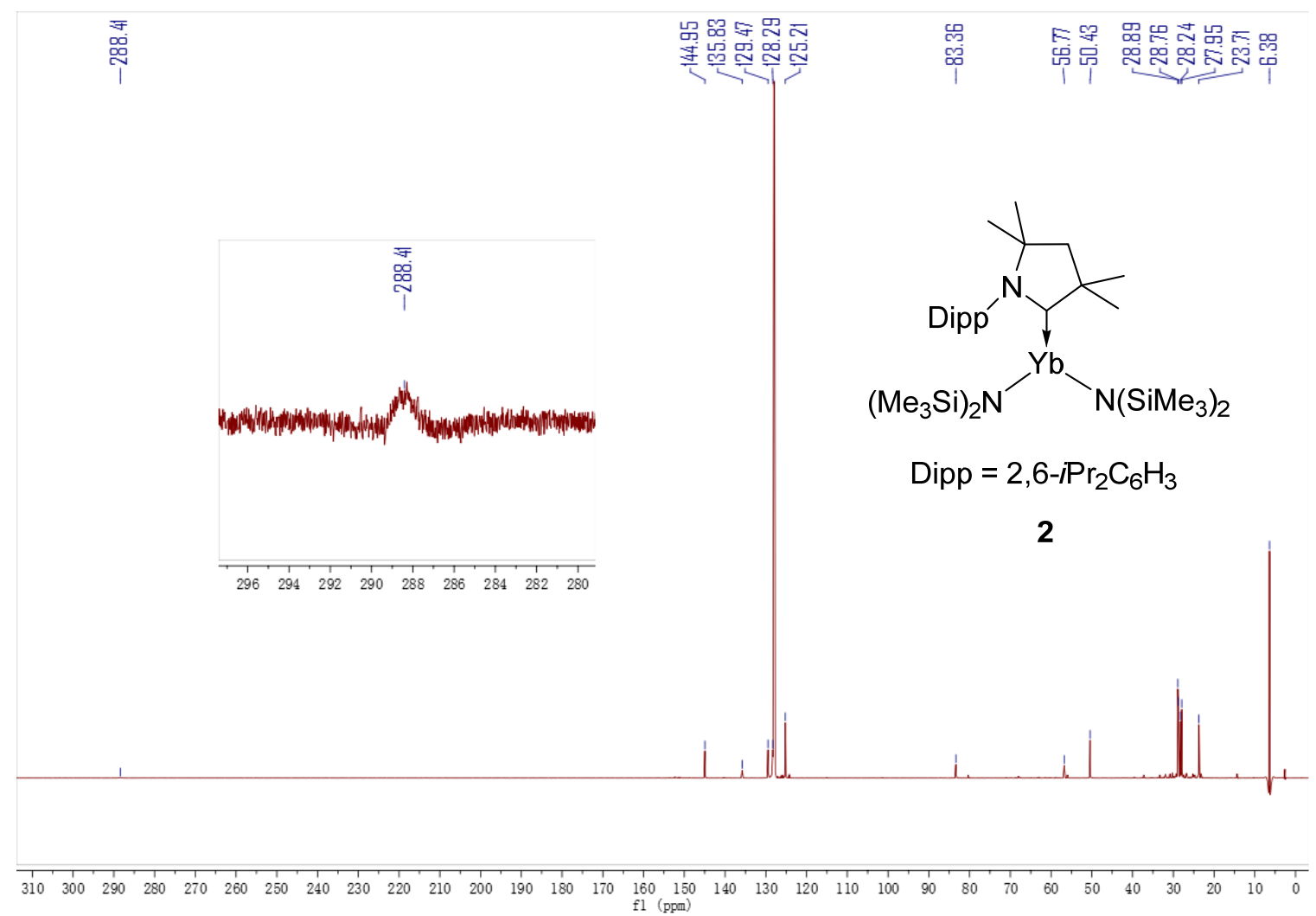

Figure S10. ${ }^{13} \mathrm{C}$ NMR spectrum of 2 in $\mathrm{C}_{6} \mathrm{D}_{6}(298 \mathrm{~K})$. 


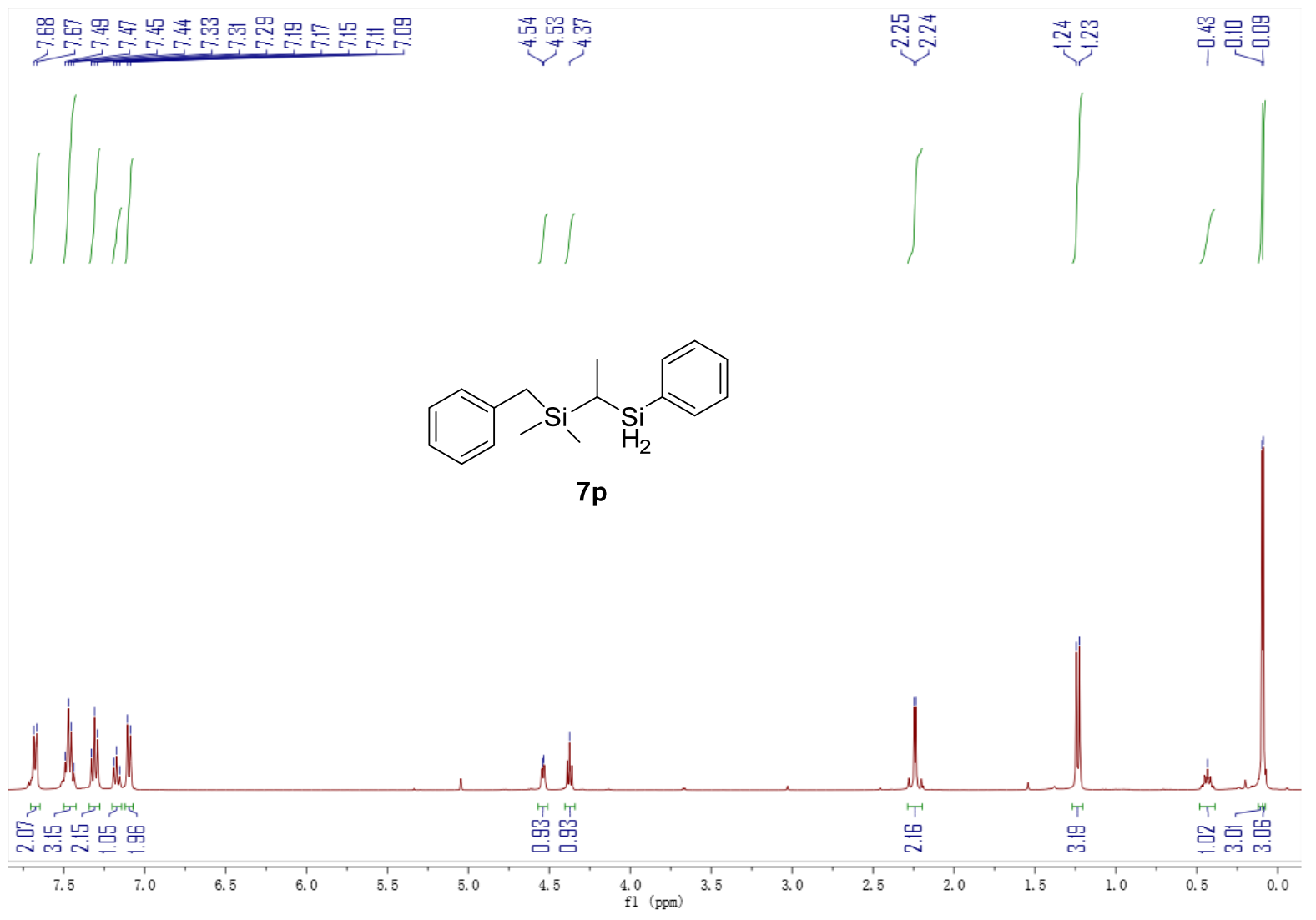

Figure S11. ${ }^{1} \mathrm{H}$ NMR spectrum of $\mathbf{7 p}$ in $\mathrm{CDCl}_{3}(298 \mathrm{~K})$.

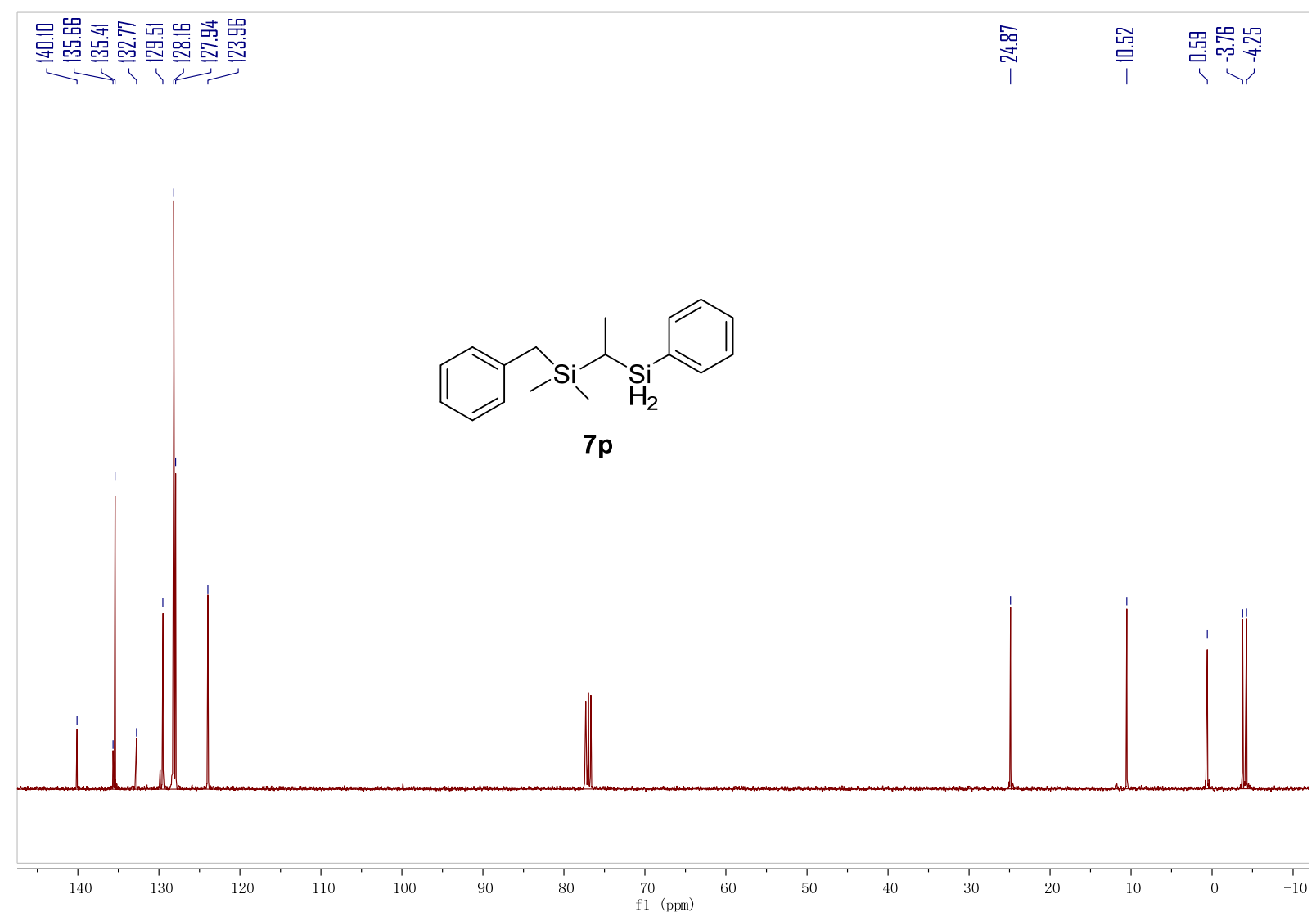

Figure S12. ${ }^{13} \mathrm{C}$ NMR spectrum of $\mathbf{7 p}$ in $\mathrm{CDCl}_{3}(298 \mathrm{~K})$. 


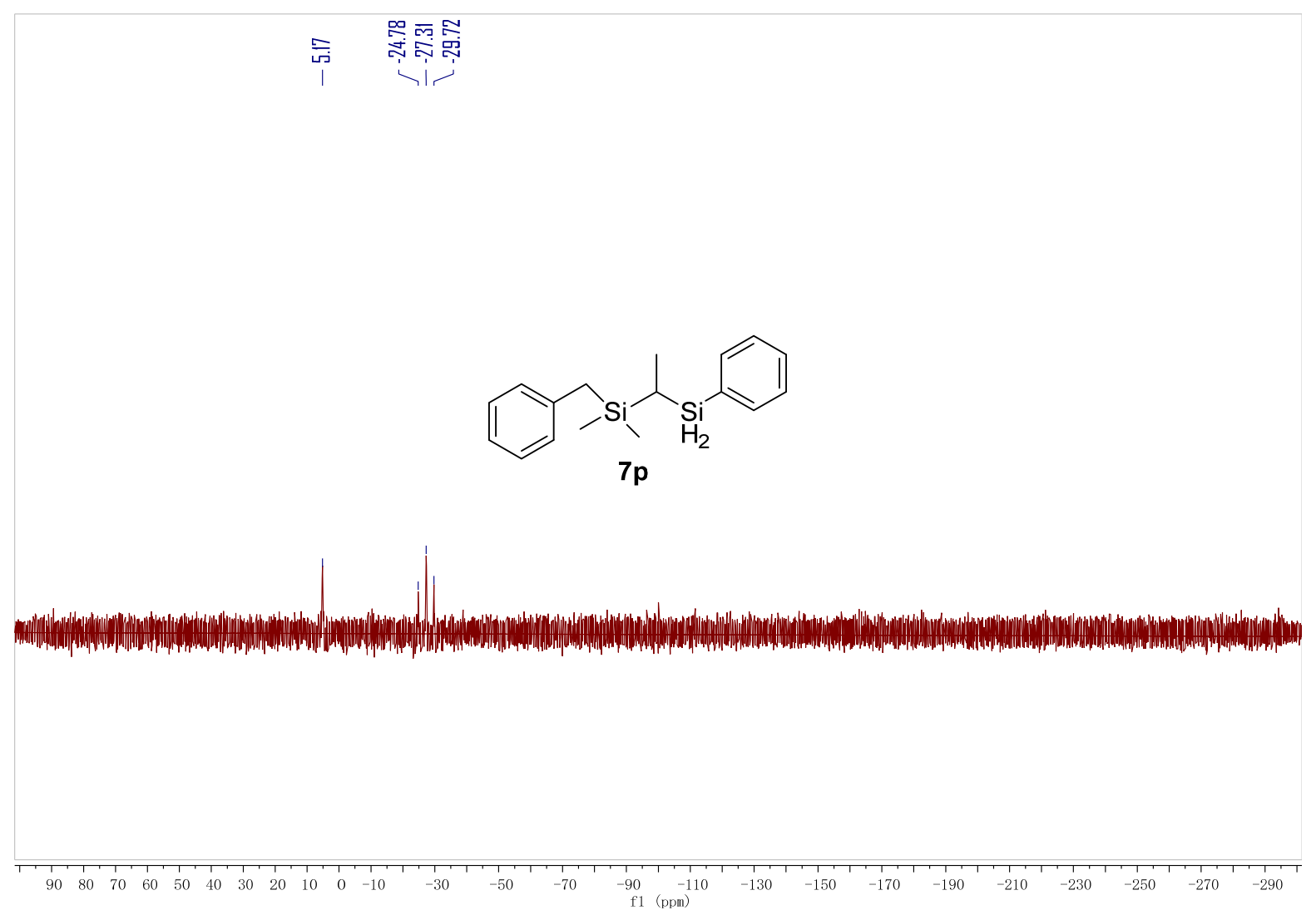

Figure S13. proton-coupled ${ }^{29} \mathrm{Si}$ NMR spectrum of $\mathbf{7 p}$ in $\mathrm{CDCl}_{3}(298 \mathrm{~K})$.

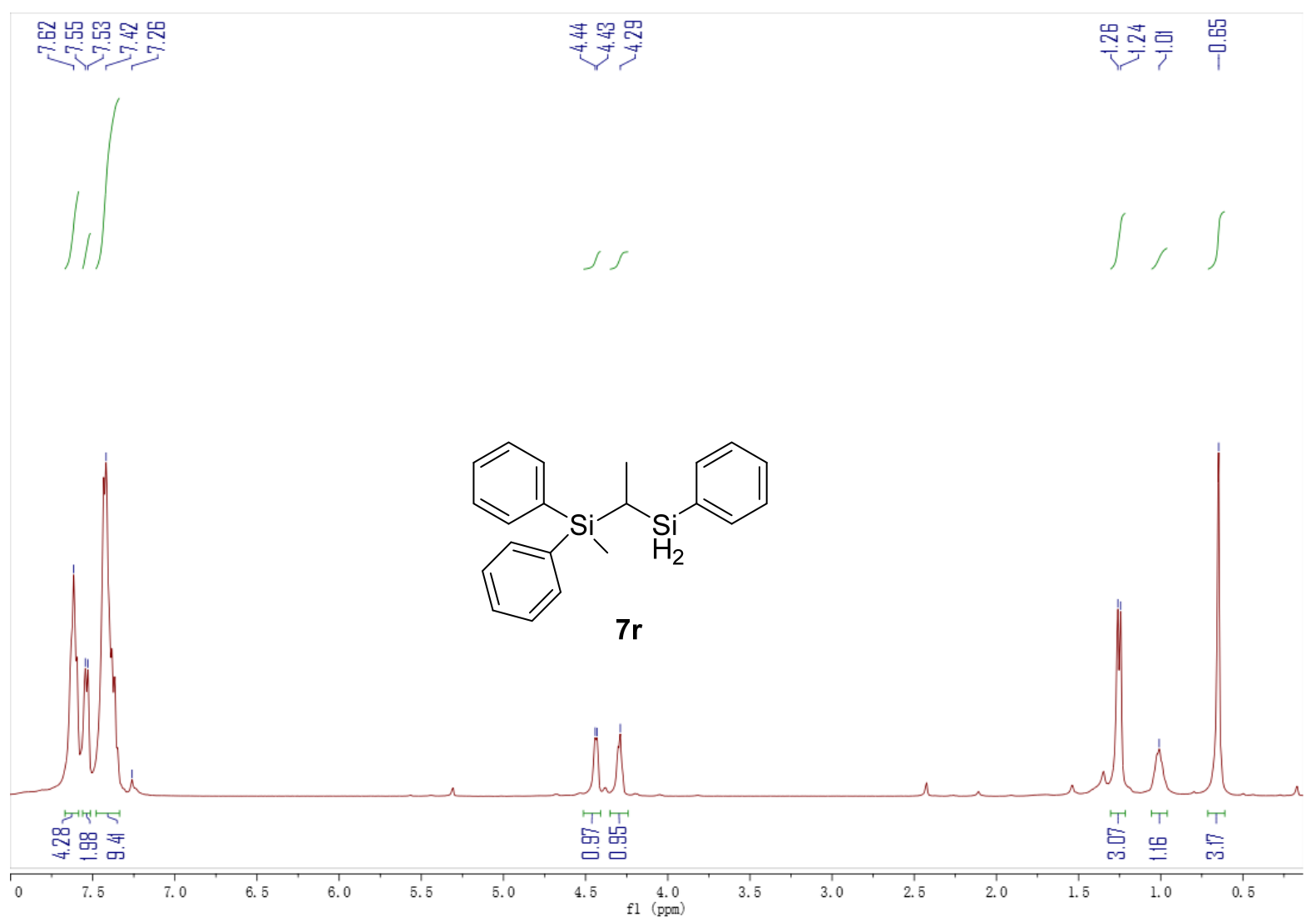

Figure S14. ${ }^{1} \mathrm{H}$ NMR spectrum of 7r in $\mathrm{CDCl}_{3}(298 \mathrm{~K})$. 


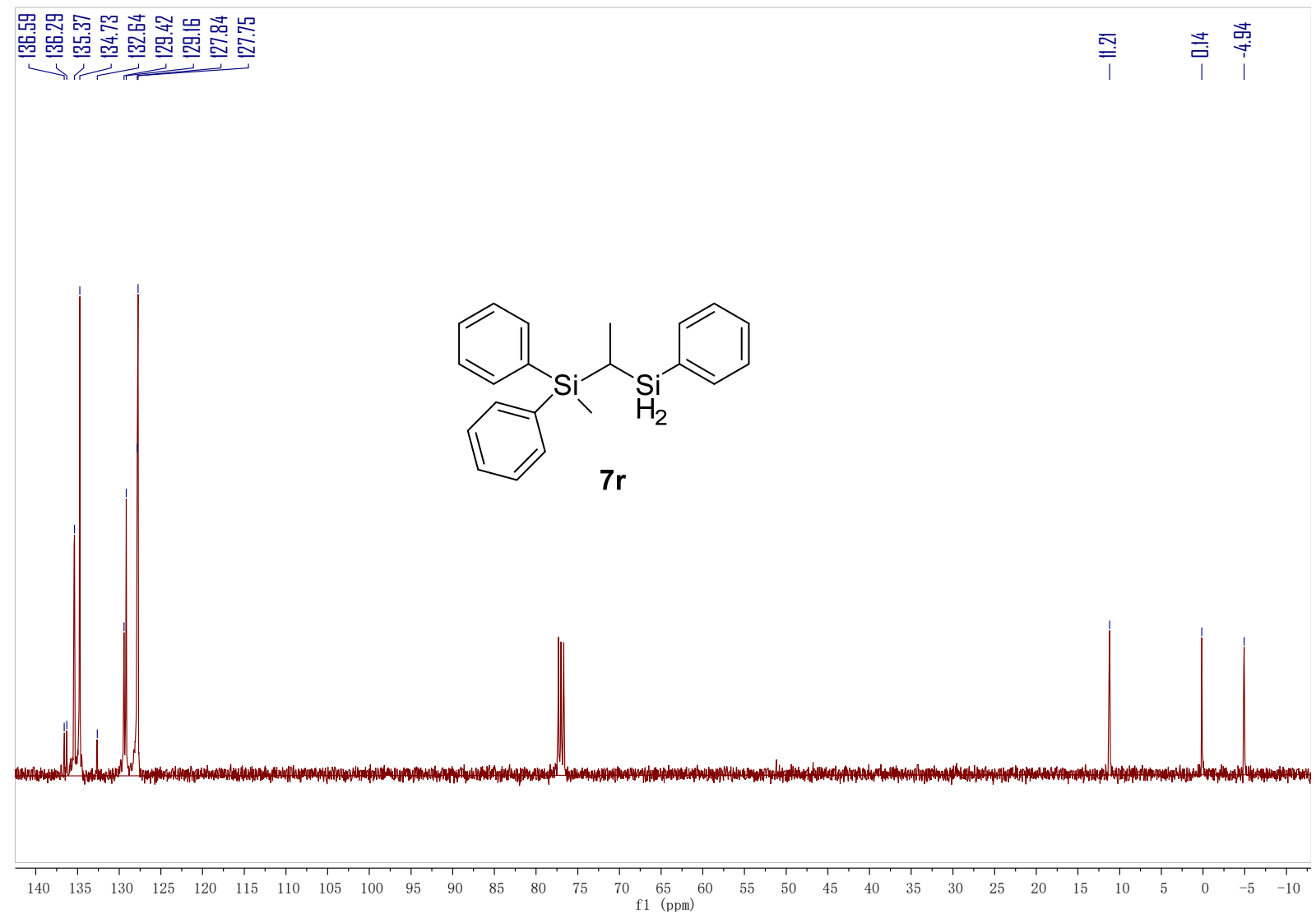

Figure S15. ${ }^{13} \mathrm{C}$ NMR spectrum of $7 \mathbf{r}$ in $\mathrm{CDCl}_{3}(298 \mathrm{~K})$.

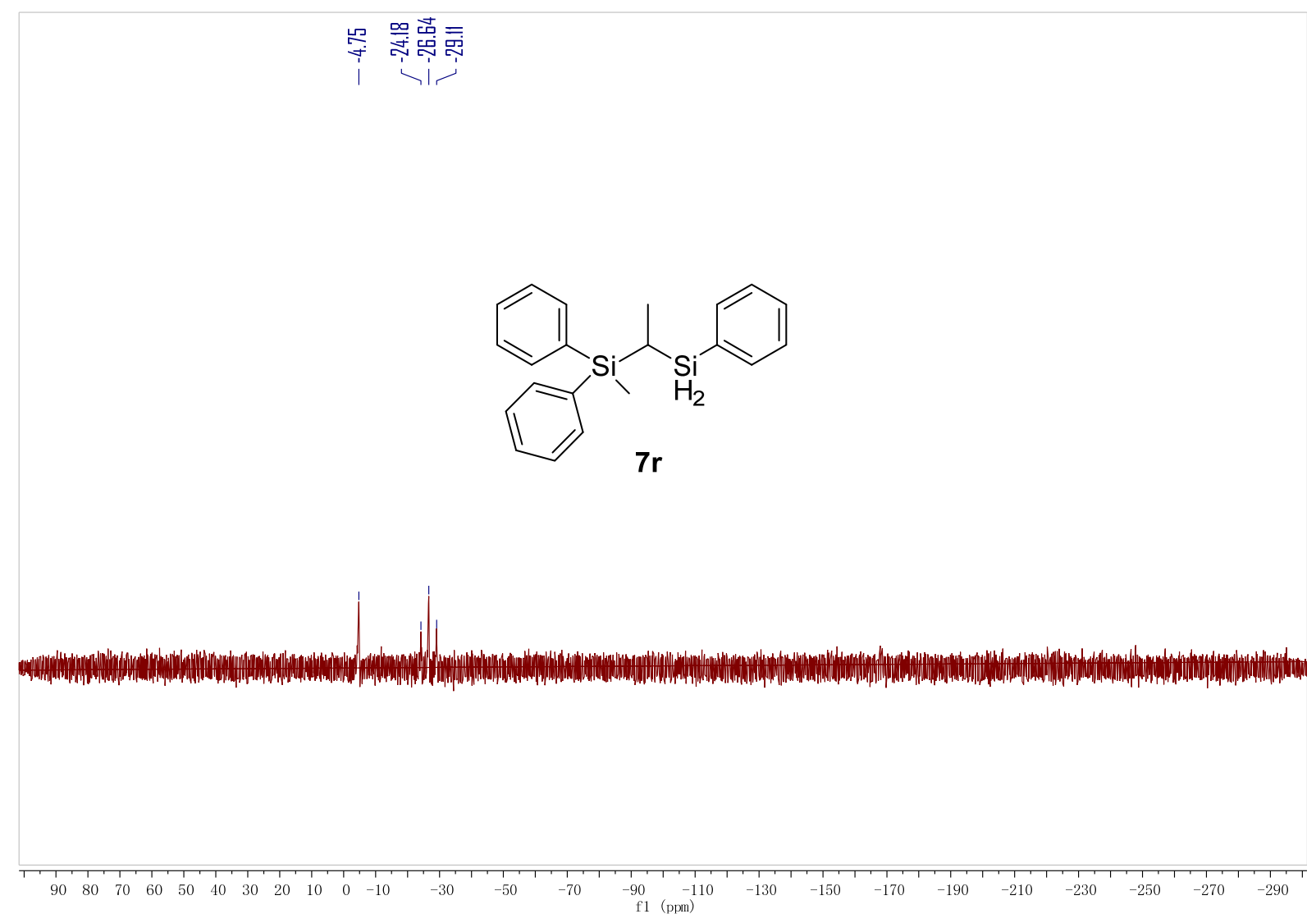

Figure S16. proton-coupled ${ }^{29} \mathrm{Si} \mathrm{NMR} \mathrm{spectrum} \mathrm{of} 7 \mathbf{r}$ in $\mathrm{CDCl}_{3}(298 \mathrm{~K})$. 


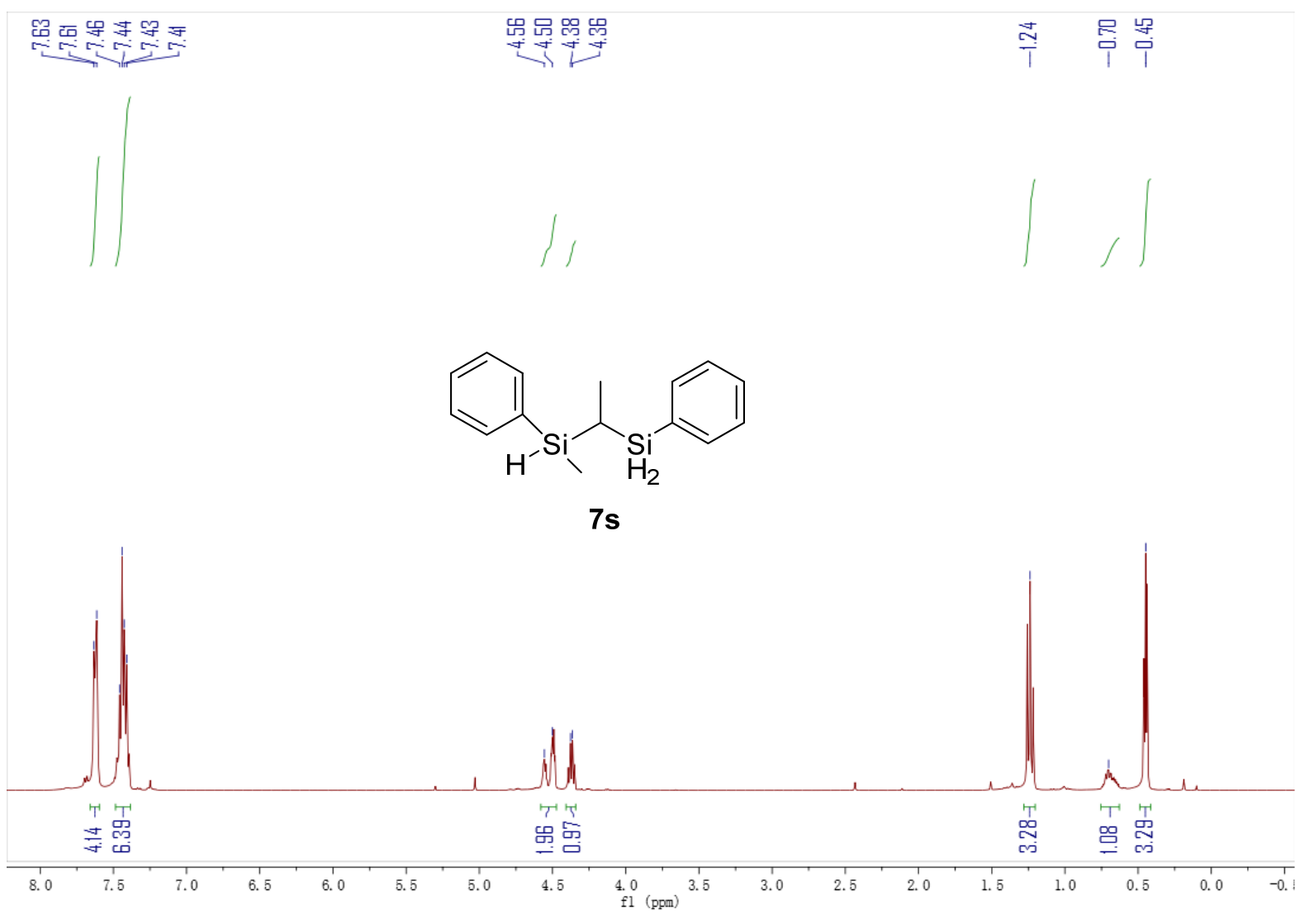

Figure S17. ${ }^{1} \mathrm{H}$ NMR spectrum of $7 \mathbf{s}$ in $\mathrm{CDCl}_{3}(298 \mathrm{~K})$.

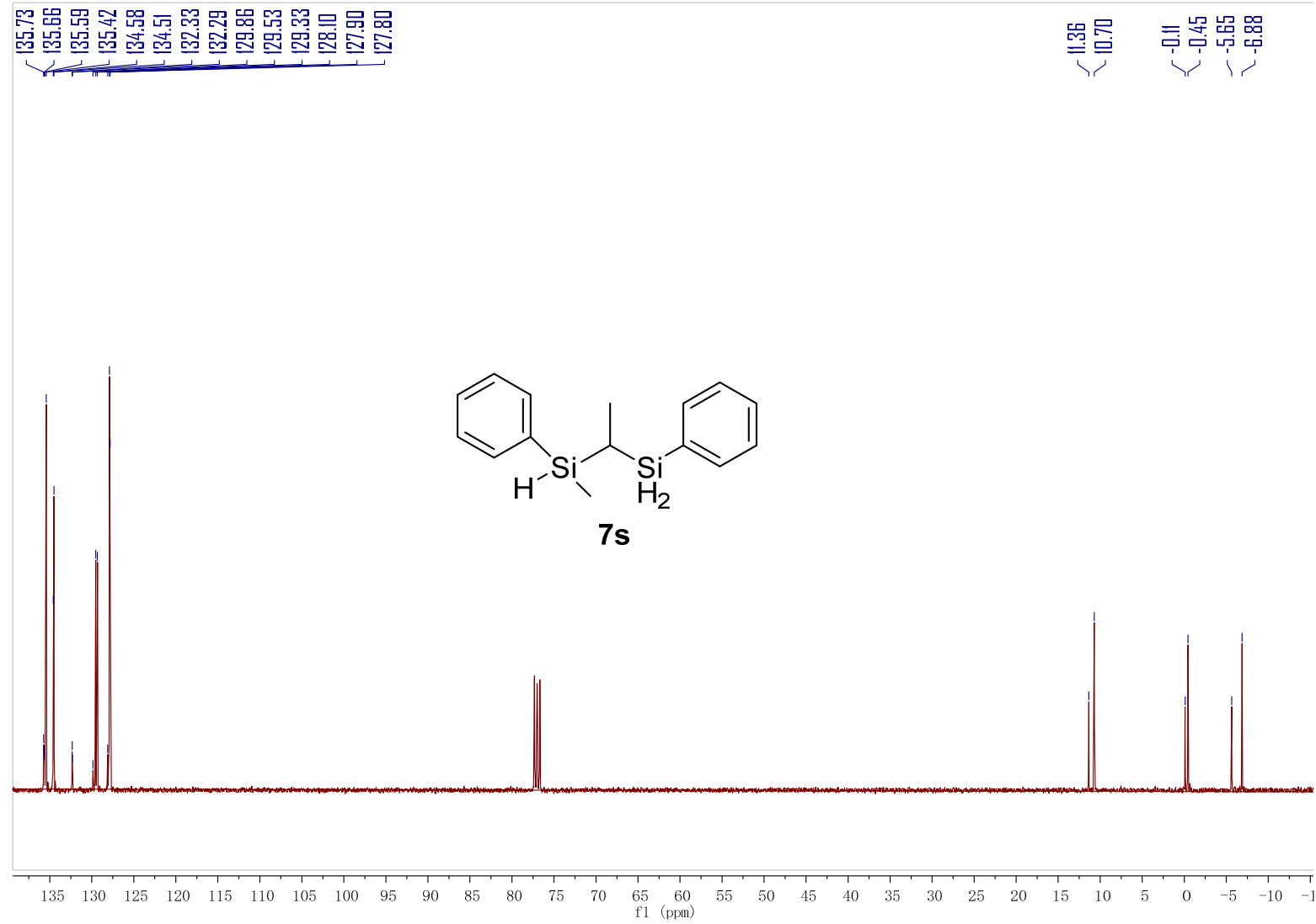

Figure S18. ${ }^{13} \mathrm{C}$ NMR spectrum of 7s in $\mathrm{CDCl}_{3}(298 \mathrm{~K})$. 


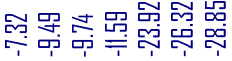

ij<smiles>CC([SiH2]c1ccccc1)[Si](C)(C)c1ccccc1</smiles>

$7 s$

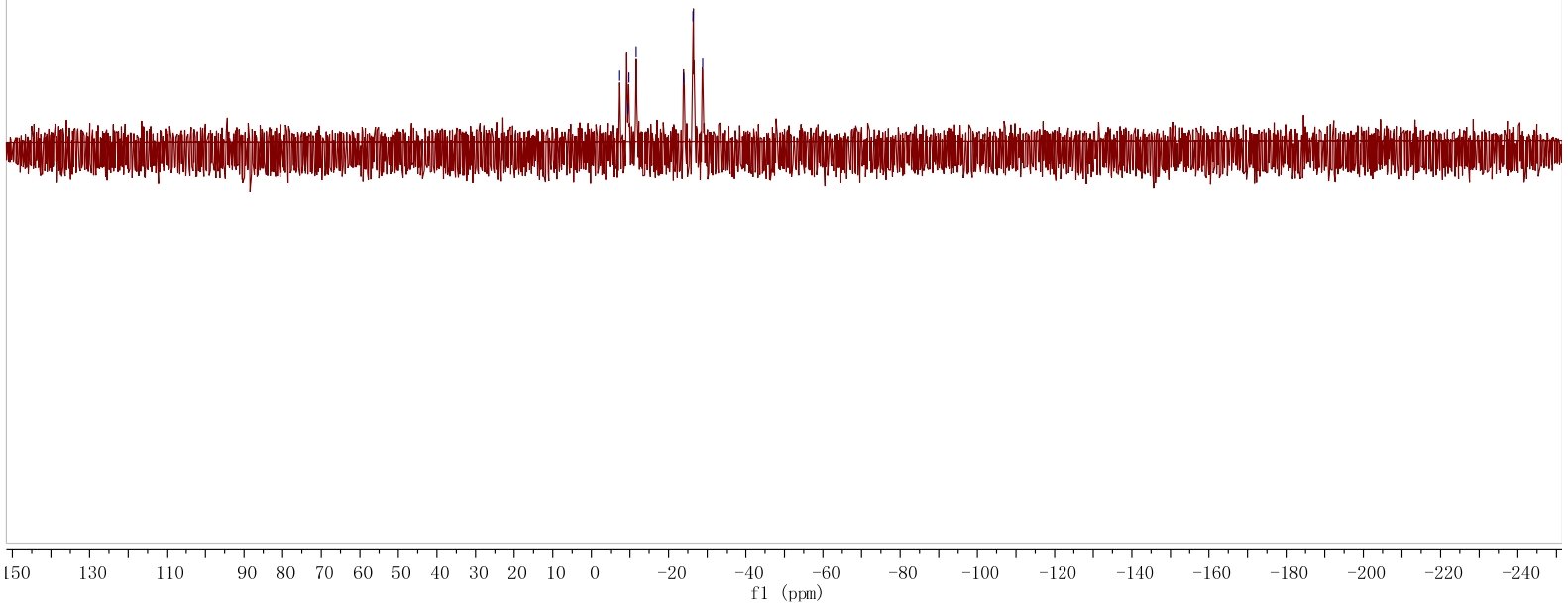

Figure S19. proton-coupled ${ }^{29} \mathrm{Si} \mathrm{NMR} \mathrm{spectrum} \mathrm{of} \mathrm{7s} \mathrm{in} \mathrm{CDCl}_{3}(298 \mathrm{~K})$.

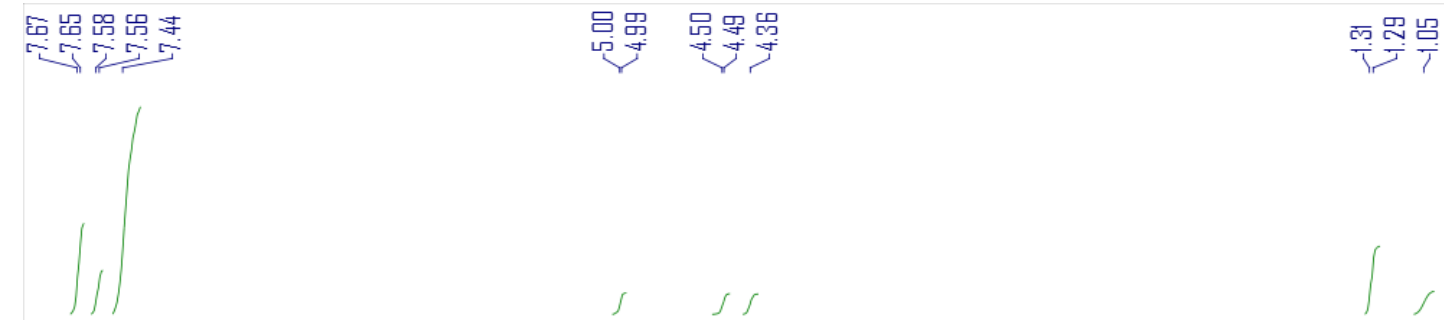<smiles>CC([SiH2]c1ccccc1)[SiH](c1ccccc1)c1ccccc1</smiles>

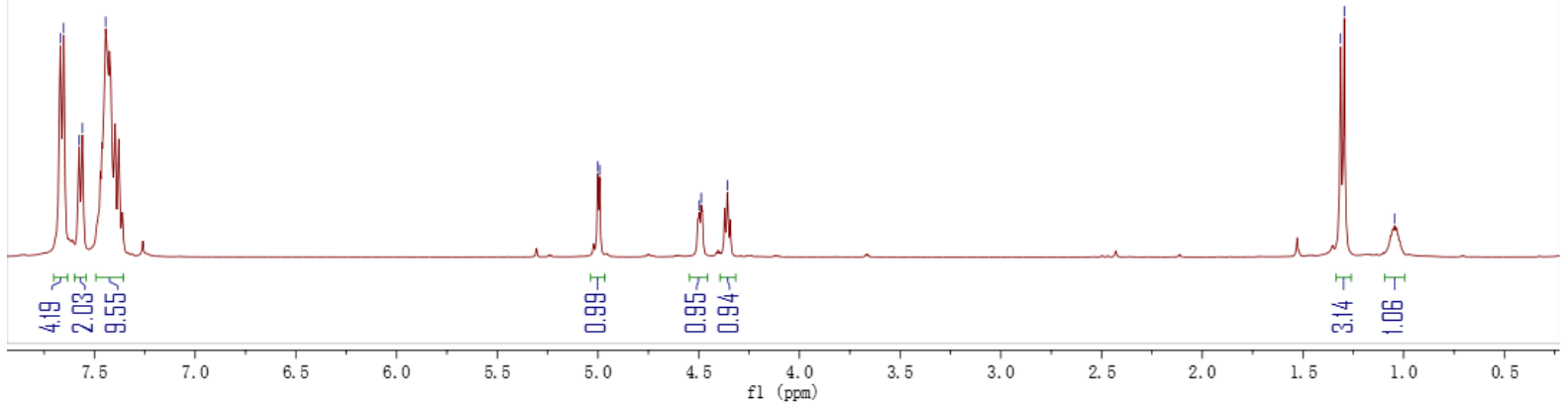

Figure S20. ${ }^{1} \mathrm{H}$ NMR spectrum of $\mathbf{7 t}$ in $\mathrm{CDCl}_{3}(298 \mathrm{~K})$. 

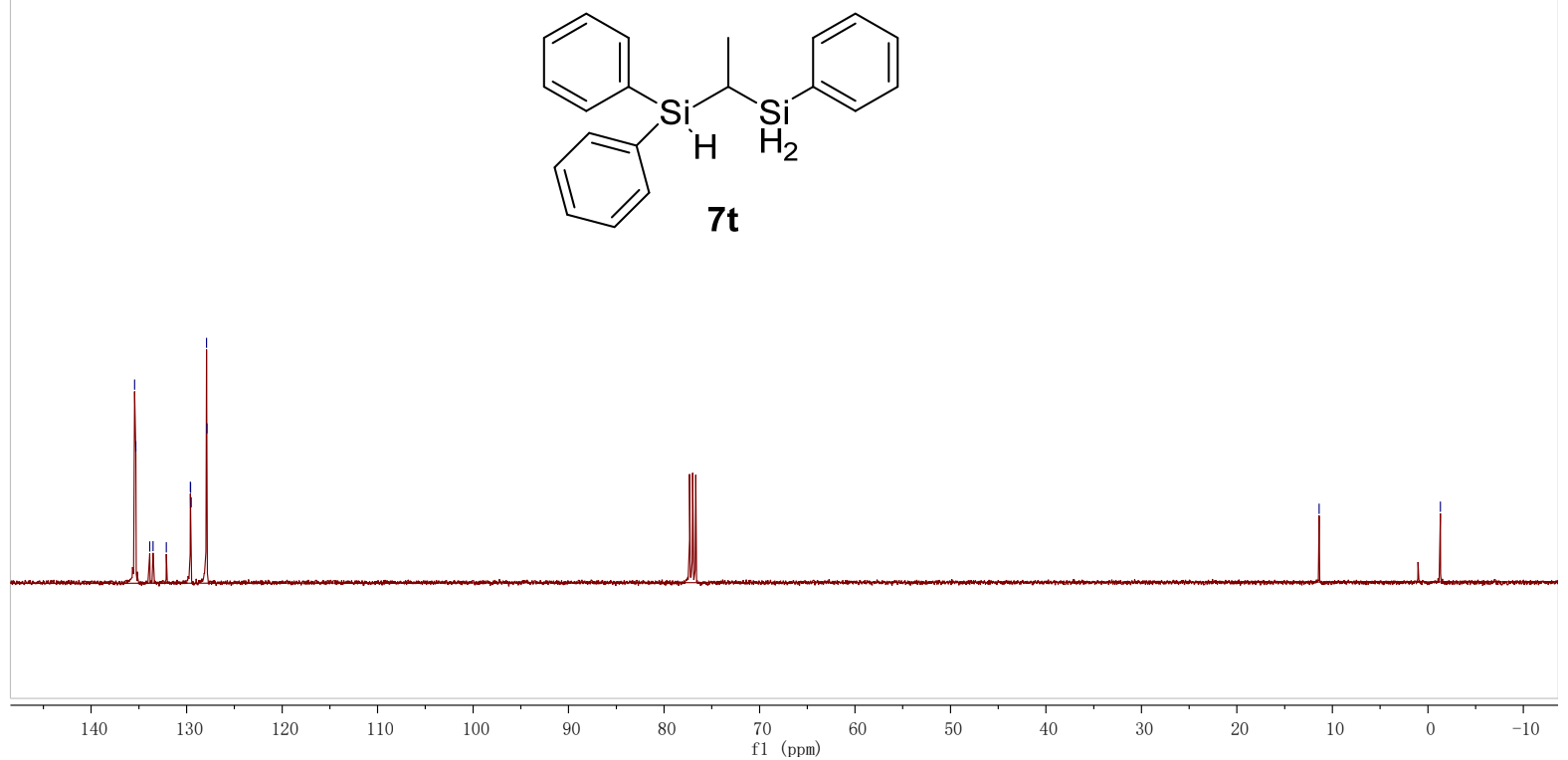

Figure S21. ${ }^{13} \mathrm{C}$ NMR spectrum of $7 \mathbf{t}$ in $\mathrm{CDCl}_{3}(298 \mathrm{~K})$.

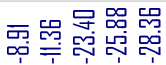

i i i

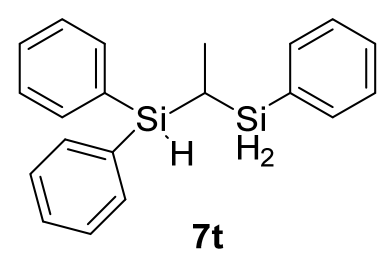

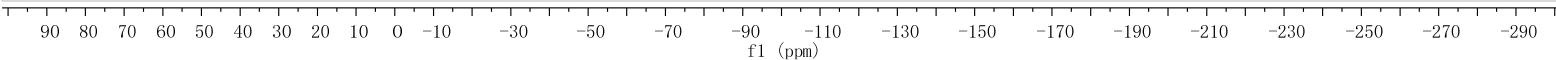

Figure S22. proton-coupled ${ }^{29} \mathrm{Si}$ NMR spectrum of $\mathbf{7 t}$ in $\mathrm{CDCl}_{3}(298 \mathrm{~K})$. 


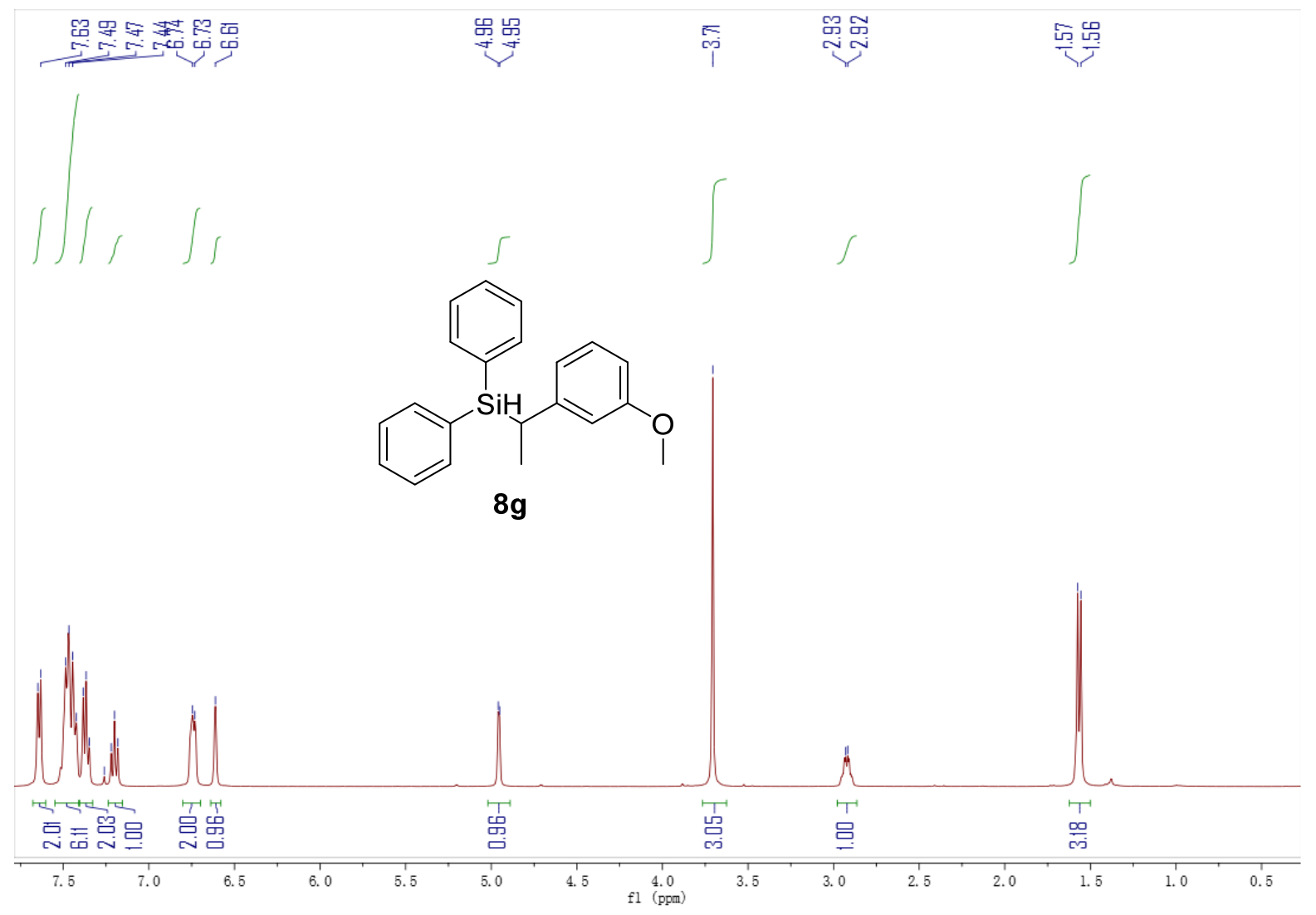

Figure S23. ${ }^{1} \mathrm{H}$ NMR spectrum of $\mathbf{8 g}$ in $\mathrm{CDCl}_{3}(298 \mathrm{~K})$.

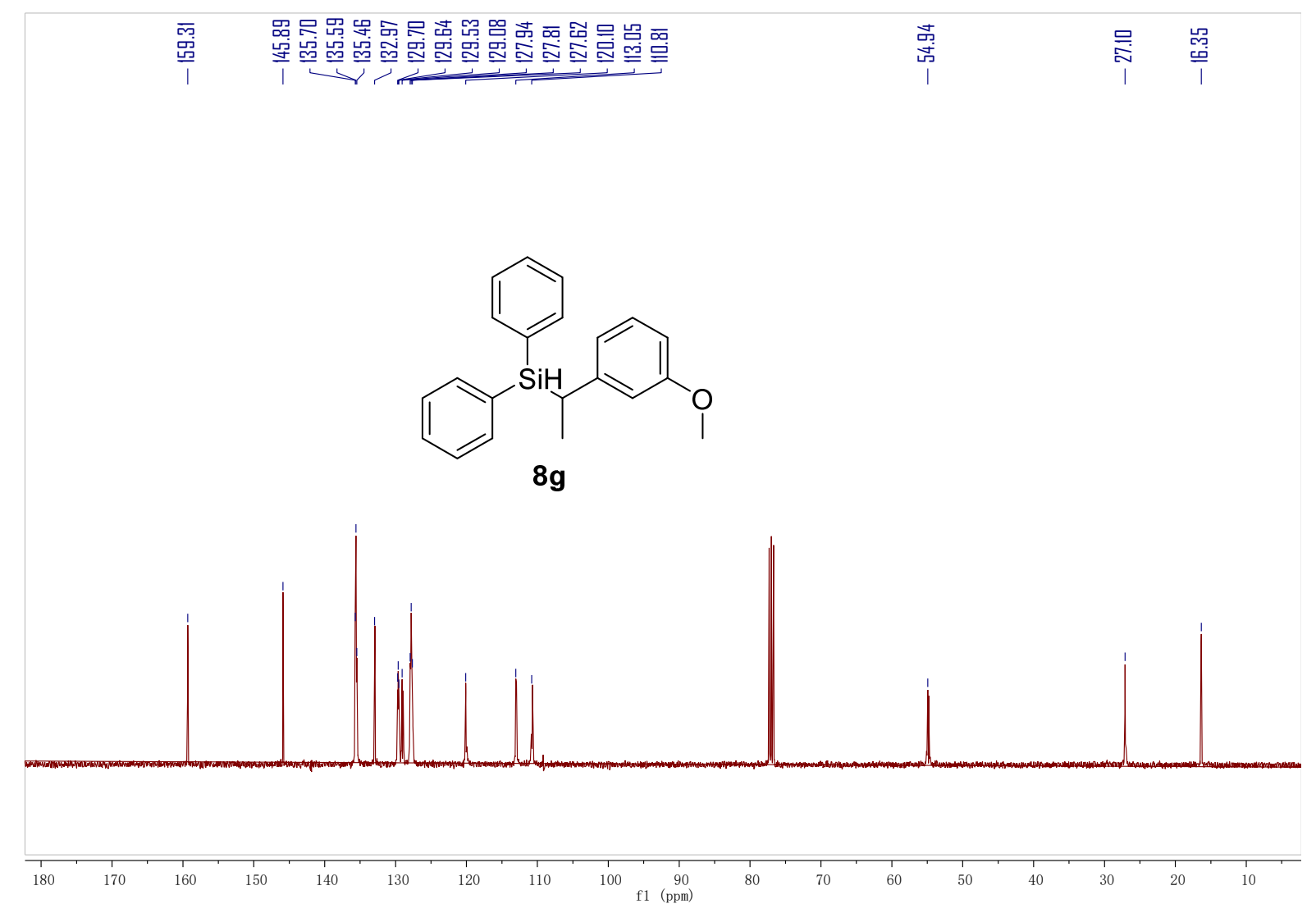

Figure S24. ${ }^{13} \mathrm{C}$ NMR spectrum of $\mathbf{8 g}$ in $\mathrm{CDCl}_{3}(298 \mathrm{~K})$. 


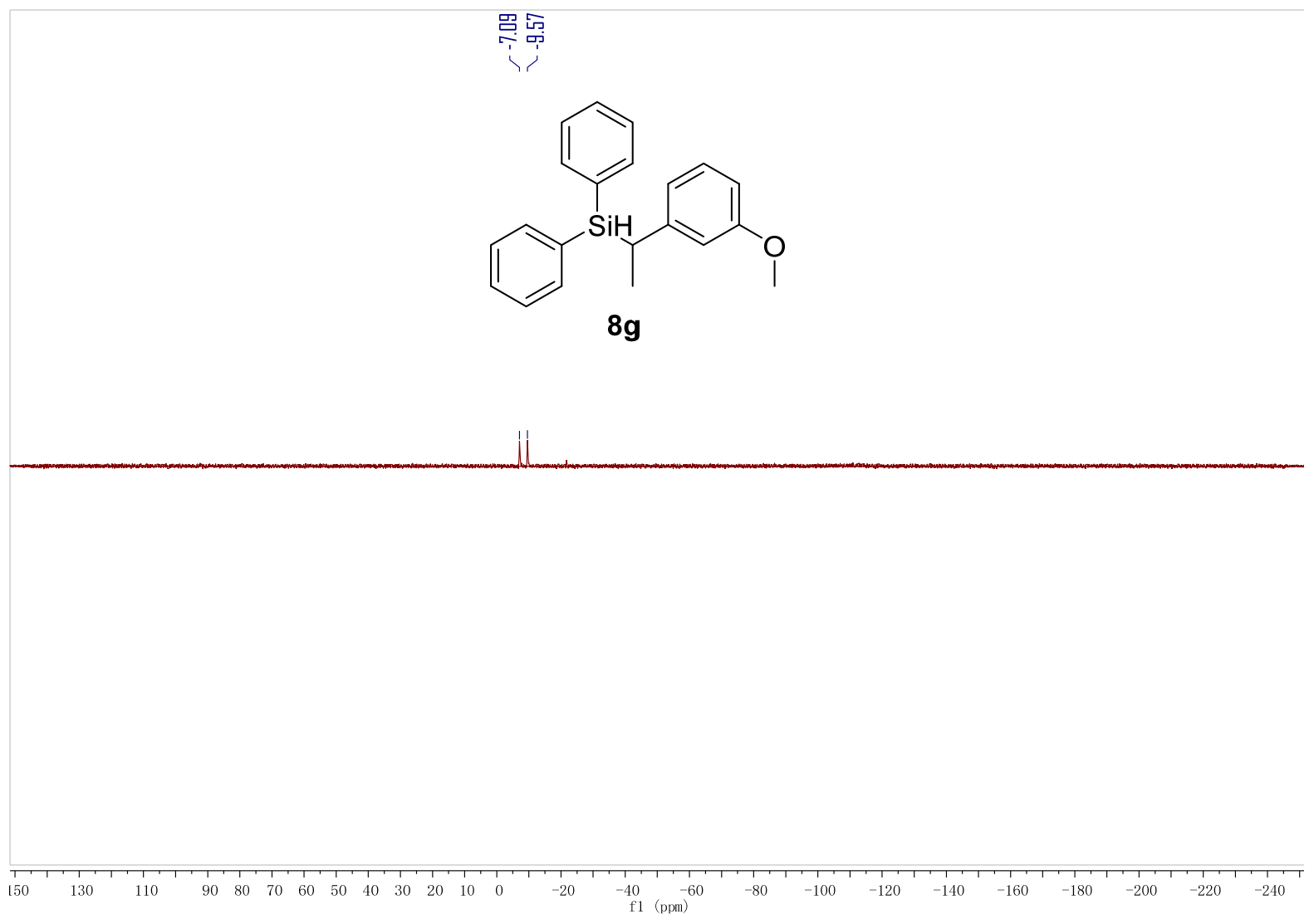

Figure S25. proton-coupled ${ }^{29} \mathrm{Si} \mathrm{NMR} \mathrm{spectrum} \mathrm{of} \mathbf{8 g}$ in $\mathrm{CDCl}_{3}(298 \mathrm{~K})$.
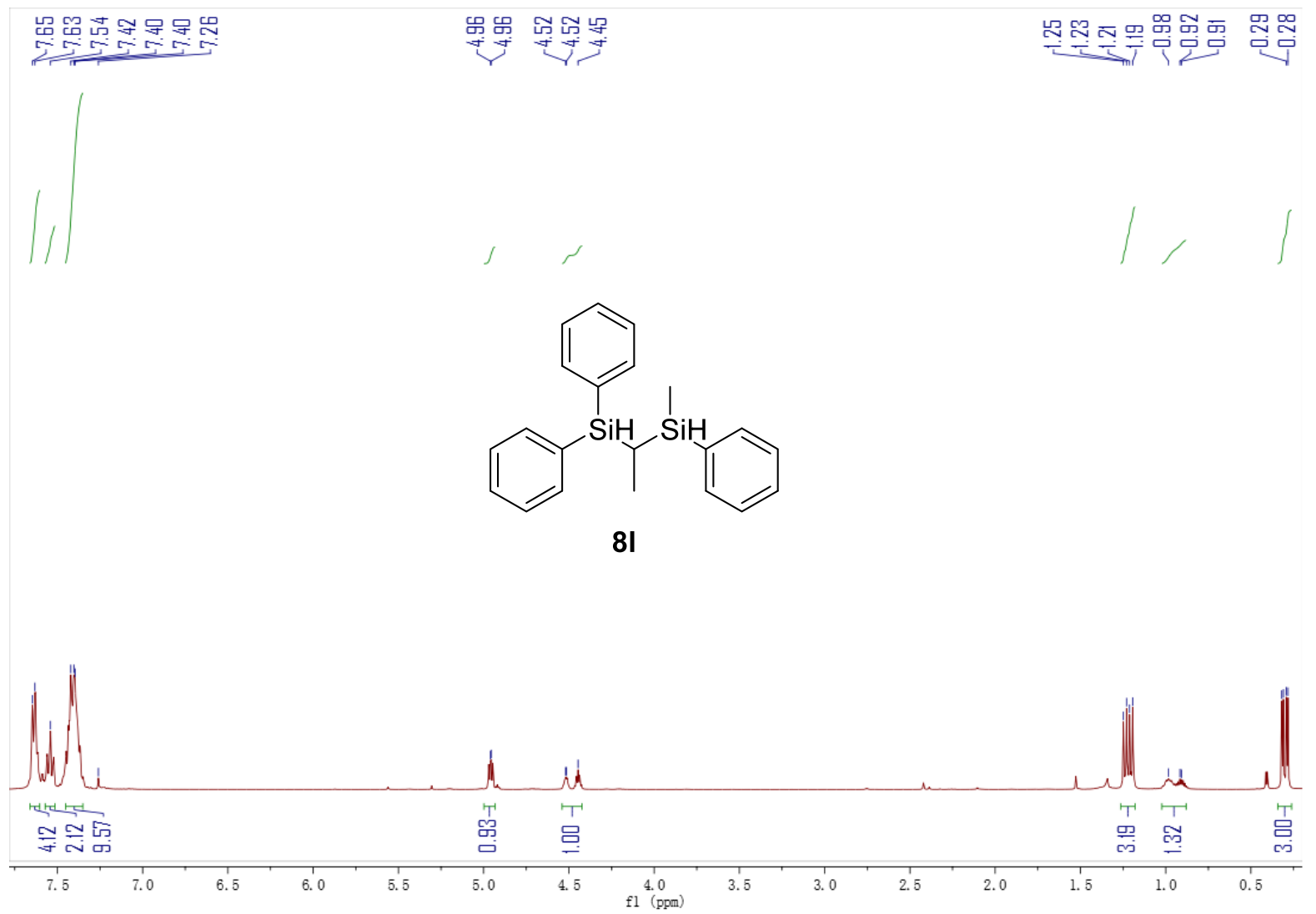

Figure S26. ${ }^{1} \mathrm{H}$ NMR spectrum of $\mathbf{8 1}$ in $\mathrm{CDCl}_{3}(298 \mathrm{~K})$. 


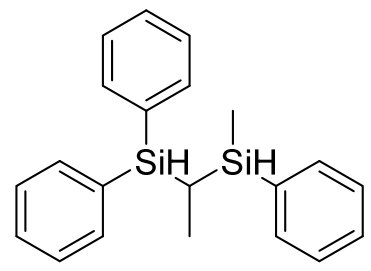

8I
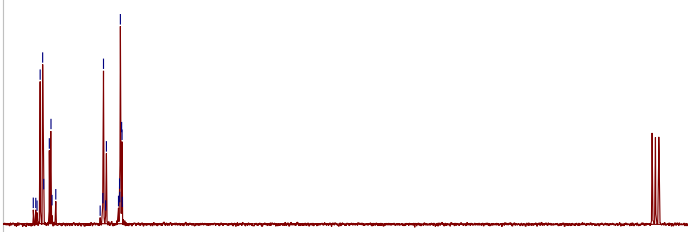

$\begin{array}{lllllllllll}135 & 130 & 125 & 120 & 115 & 110 & 105 & 100 & 95 & 90 & 8\end{array}$

Figure S27. ${ }^{13} \mathrm{C}$ NMR spectrum of $\mathbf{8 1}$ in $\mathrm{CDCl}_{3}(298 \mathrm{~K})$.

员员过<smiles>CC([SiH](C)c1ccccc1)[SiH](c1ccccc1)c1ccccc1</smiles>

8I

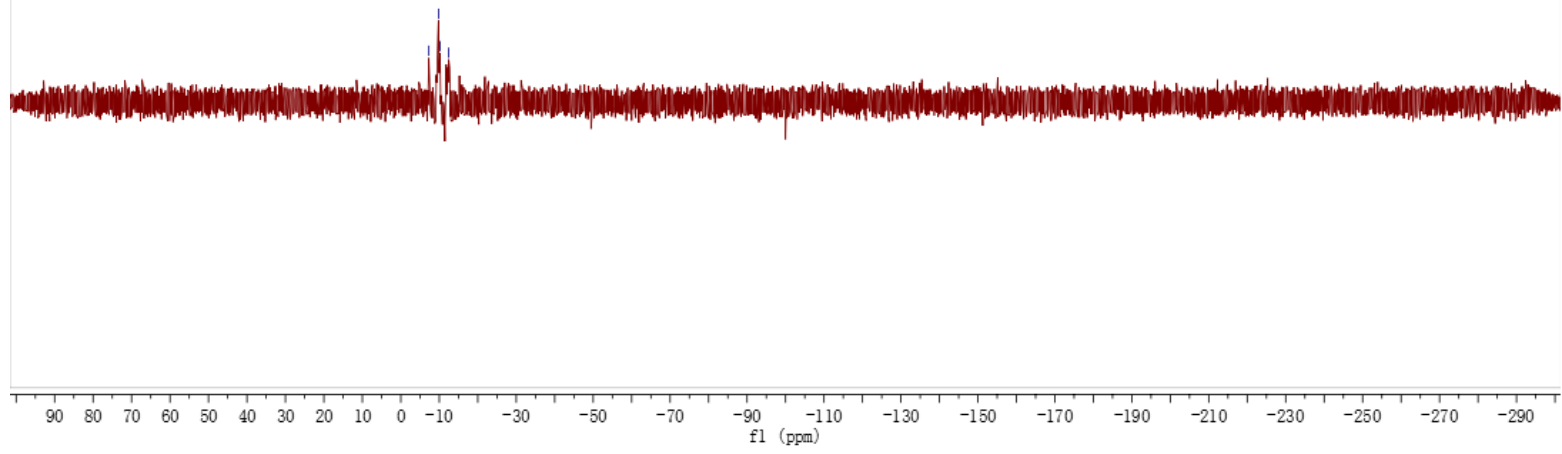

Figure S28. proton-coupled ${ }^{29} \mathrm{Si} \mathrm{NMR} \mathrm{spectrum} \mathrm{of} \mathbf{8 1}$ in $\mathrm{CDCl}_{3}(298 \mathrm{~K})$. 